\title{
LICK INDICES IN THE THIN AND THICK DISKS OF EDGE-ON DISK GALAXIES
}

\author{
Peter Yoachim ${ }^{1,2}$ and Julianne J. Dalcanton ${ }^{2,3}$ \\ Received 2008 March 31; accepted 2008 May 20
}

\begin{abstract}
We have measured Lick index equivalent widths to derive luminosity weighted stellar ages and metallicities for thin- and thick-disk-dominated regions of nine edge-on disk galaxies with the ARC $3.5 \mathrm{~m}$ telescope at Apache Point Observatory. In all cases, the thick disks are confirmed to be old stellar populations, with typical ages between 4 and $10 \mathrm{Gyr}$. The thin disks are uniformly younger than the thick disks, and show strong radial age gradients, with the outer regions of the disks being younger than 1 Gyr. We do not detect any significant metallicity differences or $\alpha$-element enhancement in the thick-disk stars compared to the thin disk, due to the insensitivity of the Lick indices to these differences at low metallicity. We compare these results to thick disks measured in other systems and to predictions from thick-disk formation models.
\end{abstract}

Subject headings: galaxies: spiral — galaxies: stellar content — galaxies: structure

Online material: color figures

\section{INTRODUCTION}

Old stellar populations preserve a fossil record of a galaxy's early formation and evolution. In the Milky Way, the stellar thick disk and halo have been recognized as the oldest stellar components and have been studied extensively. Stars in the Milky Way (MW) thick disk are old ( $\sim-12$ Gyr), and are metal poor when compared to local thin-disk stars (Reid \& Majewski 1993; Chiba $\&$ Beers 2000). Their chemical composition shows that they are enhanced in $\alpha$-elements compared to thin-disk stars, suggesting a rapid formation timescale ( $<1 \mathrm{Gyr}$ ) (Reddy et al. 2006; Brewer \& Carney 2006; Bensby et al. 2003, 2005; Prochaska et al. 2000).

Because stellar thick disks and halos are intrinsically low surface brightness features, they have been observed in detail in only a handful of galaxies. Thick disks have been photometrically detected as an excess flux at large galactic latitudes across a range of Hubble types (Dalcanton \& Bernstein 2002; Burstein 1979; Tsikoudi 1979; de Grijs \& van der Kruit 1996; de Grijs \& Peletier 1997; Pohlen et al. 2004; van der Kruit 1984; Shaw \& Gilmore 1989; van Dokkum et al. 1994; Morrison et al. 1997; Wu et al. 2002; Abe et al. 1999; Neeser et al. 2002), as well as detected in star counts using resolved stellar populations from HST (Seth et al. 2005; Mould 2005; Tikhonov et al. 2005; Tikhonov \& Galazutdinova 2005, 2008).

Three general classes of formation mechanisms have been put forward to explain thick disks. In the first, a previously thin disk is kinematically heated. In this scenario, stars form in a thin disk and increase their velocity dispersion with time. This vertical heating can be rapid, due to interactions and mergers (Quinn et al. 1993; Walker et al. 1996; Velazquez \& White 1999; Chen et al. 2001; Robin et al. 1996; Villalobos \& Helmi 2008) or gradual due to scattering off giant molecular clouds, spiral arms, and/or dark matter substructure (Villumsen 1985; Hänninen \& Flynn 2002; Benson et al. 2004; Hayashi \& Chiba 2006; Kazantzidis et al. 2007). In the second formation scenario, stars "form thick" with star formation occurring above the midplane of the galaxy

\footnotetext{
1 Department of Astronomy and McDonald Observatory, University of Texas, Austin, TX 78712; yoachim@astro.as.utexas.edu.

2 Department of Astronomy, University of Washington, Box 351580, Seattle WA, 98195.

3 Tom and Margo Wyckoff Fellow.
}

(Brook et al. 2004) or form with large initial velocity dispersions in massive stellar clusters (Kroupa 2002). In the final class of models, thick-disk stars are directly accreted from satellite galaxies. Numerical simulations have shown that stars in disrupted satellite galaxies can be deposited onto thick-disk-like orbits (Abadi et al. 2003; Martin et al. 2004; Bekki \& Chiba 2001; Gilmore et al. 2002; Navarro et al. 2004; Statler 1988; Read et al. 2008). While these models were originally developed to explain the origin of the MW thick disk, they should work equally well for thick disks in other massive galaxies.

On the other hand, some of these mechanisms are likely to be less effective in lower mass galaxies which have lower density disks, little or no spiral structure, and fewer satellites hosting stars. Lower mass galaxies also have different formation times, environments, and gravitational potentials that may also lead to variation in the mass, age, and metallicity of thick disks of low-mass galaxies.

To test these formation models, detailed comparisons of thinand thick-disk properties are required across a range of galaxy masses. In particular, the relative ages and chemical enrichment patterns of the thin and thick disks should differ among these formation models. If the thick disk is just a gradually kinematically heated thin disk, there should be a smooth age and enrichment gradient between the two. In contrast, if the thick disk is formed from accreted stars we should expect the ages and metallicities of the thin and thick disks to be only weakly correlated. We may also expect to see variations with the mass of the galaxies, with less massive galaxies being more susceptible to external heating and more massive galaxies being better able to tidally disrupt satellites.

Measuring the ages and metallicities of thick disks outside the MW has proved to be challenging. Ages and metallicities can be derived from isochrone fits to thin- and thick-disk stars resolved with $H S T$, provided that the host galaxies are sufficiently close and oriented edge-on to the line of sight. The systems studied this way show older populations at large-scale heights but little vertical metallicity gradient, at least for the low-mass galaxies $\left(V_{c}<\right.$ $100 \mathrm{~km} \mathrm{~s}^{-1}$ ) which dominate these samples (Seth et al. 2005; Tikhonov et al. 2005; Mould 2005).

For the systems that are farther away, only broadband colors have been used to estimate the ages and metallicities of the thick 
disks. When thick disks are photometrically detected, they typically have very red colors $(B-R \sim 1.3-1.5)$ (Yoachim \& Dalcanton 2006; Dalcanton \& Bernstein 2002; van Dokkum et al. 1994) suggestive of an old population. However, stellar parameters are notoriously difficult to derive from broadband colors due to the age-metallicity degeneracy in the optical colors, and a lack of IR-colors at the low surface brightnesses of the thick-disk region.

Some progress has been made in measuring the metallicity of the thin disk using high signal-to-noise emission lines (e.g., Tremonti et al. 2004; Zaritsky et al. 1994; van Zee et al. 1998), and in measuring metallicity gradients (e.g., Zaritsky et al. 1994; van Zee et al. 1998). While the emission-lines studies can constrain the total chemical enrichment a galaxy has experienced, they tell us nothing about the underlying stellar populations in the galaxies. Emission lines are also unable to provide constraints on the properties of extraplanar stellar populations, which are likely to be dominated by old, dormant stellar populations.

To better measure the ages and metallicities of thick disks in a larger sample of galaxies, we turn to the integrated spectrum of these galaxies and use the Lick/IDS absorption-line system to derive luminosity-weighted stellar population properties. The Lick indices were originally developed for studying older stellar populations (Burstein et al. 1984; Faber et al. 1985), and have been used extensively in analyzing elliptical galaxies and globular clusters (e.g., Trager et al. 1998, 2000a; Sánchez-Blázquez et al. 2007). Worthey et al. (1994) showed that using a combination of age-sensitive (Balmer lines) and metallicity-sensitive ( $\mathrm{Mg} b$ and $\mathrm{Fe}$ ) indices, one can lift the age-metallicity degeneracy for a stellar population. Stellar spectral libraries have now been used to create SSP models over a large range of metallicity and age combinations (Worthey 1994; Vazdekis \& Arimoto 1999), including models with variable $\alpha$-element enhancement (Thomas et al. 2003). The tools also now exist to calculate expected Lick indices for composite stellar populations (Bruzual \& Charlot 2003).

Despite the development of stellar synthesis codes that can be extended to younger stellar populations, relatively few studies have attempted to observe Lick indices in disk galaxies. Studies using tunable filters have been able to detect $\mathrm{Mg}$ and Fe index gradients in disks (Beauchamp \& Hardy 1997; Ryder et al. 2005), but have not been combined with measurements of age-sensitive indices. Studies of disk systems have tended to focus on the high surface brightness bulge components (Moorthy \& Holtzman 2006; Peletier et al. 2007; Prugniel et al. 2001; Proctor et al. 2000), and fail to reach very far into the disks. In the most extensive study observing Lick indices in disk galaxies, MacArthur (2006) observed Lick indices in eight galaxy disks, including several late-type galaxies out to $\sim 1$ scale length. These observations probed to $\sim 1$ scale length. However, all of these galaxies were fairly face-on, preventing thick- and thin-disk components from being separated.

In this paper we target regions of edge-on galaxies that are dominated by either the stellar thick disk and thin components. We then compare ages and metallicities derived from Lick indices both between the two components and from galaxy to galaxy.

\section{OBSERVATIONS}

The original sample of 49 galaxies was selected from the Flat Galaxy Catalog (Karachentsev et al. 1993) and imaged in $B, R$, and $K_{s}$ (Dalcanton \& Bernstein 2000). Dalcanton \& Bernstein (2002) used this imaging to demonstrate the ubiquity of thick disks around late-type galaxies. We have since used two-dimensional decompositions of the galaxy images to measure structural parameters for the thick and thin disks (Yoachim \& Dalcanton 2006).
We have also measured kinematic properties of the thick and thin disks using GMOS on the Gemini telescopes (Yoachim \& Dalcanton 2005; Yoachim \& Dalcanton 2008).

For this study, we selected a subset of galaxies where the photometric decompositions suggested we would be able to isolate thin- and thick-disk regions while obtaining adequate signal. This limits us to observing predominantly lower mass galaxies, as Yoachim \& Dalcanton (2006) found these are the galaxies with proportionally larger thick disks.

Observations were made using the Dual Imaging Spectrograph (DIS) on the ARC $3.5 \mathrm{~m}$ telescope at Apache Point Observatory (APO) between 2003 June and 2006 February during a total of 34 half-nights of observing. Although the telescope aperture is much smaller than used for previous studies ( $3.5 \mathrm{~m}$ compared to $8 \mathrm{~m})$, we were able to reach the needed signal-to-noise ratio $(\mathrm{S} / \mathrm{N})$ using a novel slit design. Spectroscopy of extended low surface brightness objects has traditionally been limited by the need to collect a large number of photons while maintaining adequate spectral resolution. However, since the Lick indices are defined at low spectral resolution $(\sim 8 \AA)$ and cover a limited wavelength range, it is possible to capture more photons by using a much wider slit to feed a spectrograph with a higher resolution grating. We therefore built a custom $5^{\prime \prime} \times 4^{\prime}$ slit to allow the largest possible amount of light to reach the detector. On the blue side, we used a grating with 1200 lines $\mathrm{mm}^{-1}$ and on the red side, 830 lines $\mathrm{mm}^{-1}$. The resulting spectral resolution was 4.9(7.1) A FWHM as measured from calibration lamps for the blue(red) chip. The blue chip gave wavelength coverage from 4390-5430 A sufficient to measure the age-sensitive $\mathrm{H} \beta$ and metallicity-sensitive $\mathrm{Mg}, \mathrm{Fe} 5270$, and Fe 5335 Lick indices. The red side covered 6140-7860 $\AA$, which allowed us to measure the strength of the $\mathrm{H} \alpha$ emission line. The CCDs were binned by 2 in the spectral direction to reduce read noise. Combining a large-aperture slit with a high-resolution grating is an unconventional setup, but the increased throughput of the wide slit allows us to observe the low surface brightness regions where the thick disk dominates.

The slit camera on DIS allowed us to accurately place the slit by centroiding nearby bright stars. The slit camera has a platescale of $0.298^{\prime \prime} \mathrm{pixel}^{-1}$, and we were typically able to place the slit within 1 pixel of our target region. Slit camera images were taken throughout long exposures to ensure accurate tracking. For each galaxy, we gathered spectra at the midplane as well as at several vertical scale heights above the midplane where photometric decompositions imply the majority of flux should be supplied by the thick-disk component. Images of the slit placement are shown in Figure 1 and the observation log is listed in Table 1.

We found that there were several prominent skylines near the relevant indices that would slowly fade for several hours after sunset. Most problematic was a skyline of $\mathrm{OH}$ at $5200 \AA$, which often contaminated the $\mathrm{Mg} b$ index. To avoid the skylines, we took advantage of the APO $3.5 \mathrm{~m}$ scheduling system which allocates observations in half-night intervals and scheduled most of our observations for the second half of the night.

\section{DATA REDUCTION}

Data were processed with standard IRAF/PyRAF routines along with several custom scripts written in IDL. Reduction steps for the red and blue side were identical unless otherwise noted. All the frames were bias corrected by subtracting the mean from the overscan region. Any residual bias structure was removed by subtracting a bias frame constructed from 5-10 bias exposures taken every night. For one-half night (2004 February 11 observations of FGC 913), the blue chip suffered from $60 \mathrm{~Hz}$ noise, which resulted in diagonal streaks across the images. This pattern was 

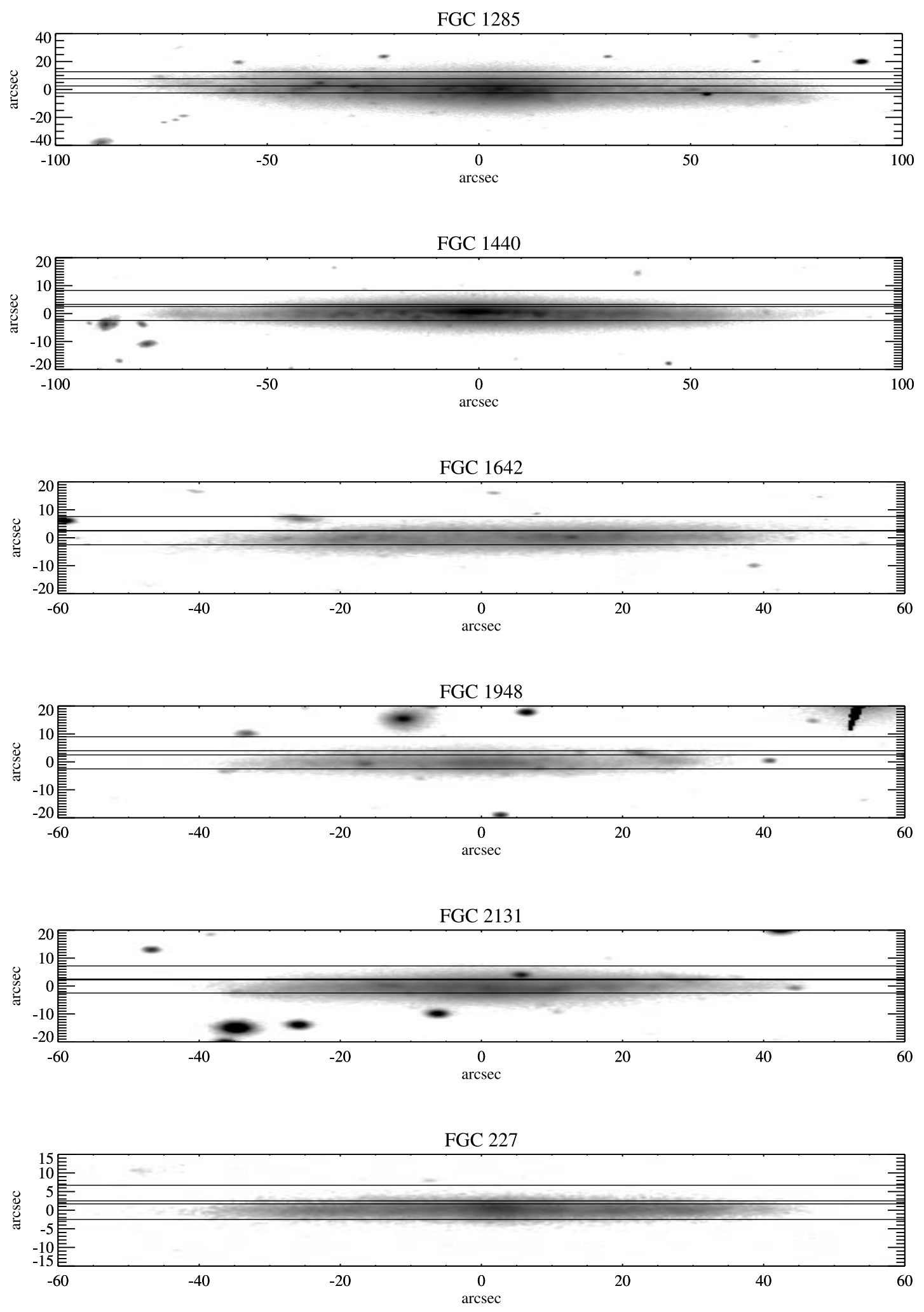

FIG. 1. $-R$-band images of our galaxy sample with the APO long-slit positions overlayed. All images are stretched to include $20<\mu_{R}<24.2 \mathrm{mag} \operatorname{arcsec}^{-2}$.

removed by shifting and subtracting a single bias frame that had similar variation in the readout pattern. The images were flatfielded using spectra of a quartz lamp. Spectra of the twilight sky showed no need for an illumination correction. The spectra were wavelength calibrated using $\mathrm{He}, \mathrm{Ne}$, and $\mathrm{Ar}$ arc lamps along with night-sky emission lines (Osterbrock et al. 1996). Arc spectra were taken interspersed with the science exposures throughout the night, approximately every hour. Stellar spectra were used to correct the spatial distortions (tilt) in the observations. Observations of standard stars and standard atmospheric extinction curves were used to flux calibrate the spectra. Few of the observations were made in photometric conditions, thus our flux calibration is 

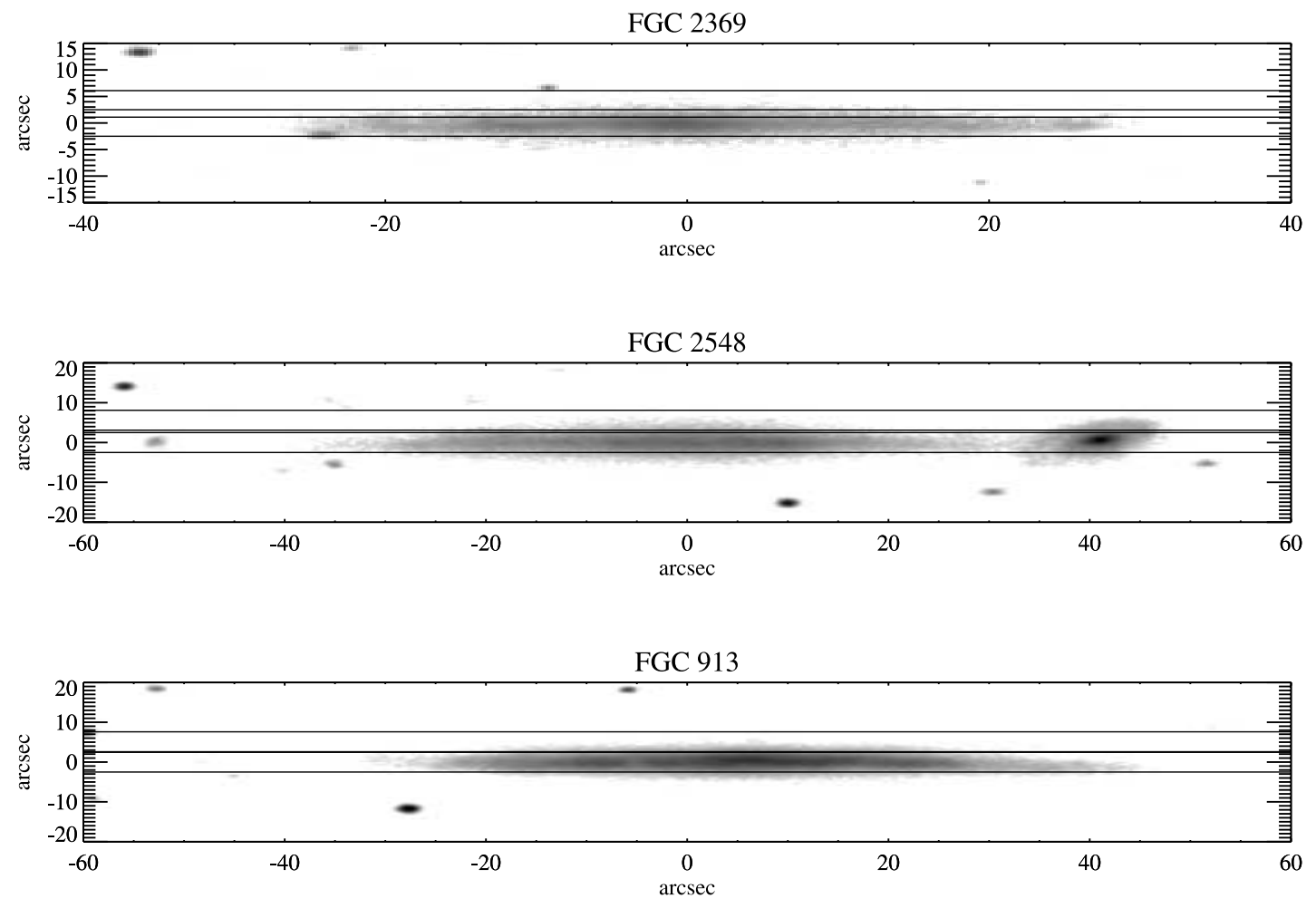

Fig. 1-Continued

primarily used as a first-order removal of the instrumental sensitivity profile. Because we are primarily interested in measuring equivalent widths, the exact flux normalization is not crucial. The sky was subtracted using a second-order polynomial fit to regions dominated by the sky. The spectra were finally corrected for motion relative to the local standard of rest, scaled to a common flux level, spatially aligned, and combined rejecting cosmic-ray hits.

Our final spectra have a spatial scale of $0.42 \operatorname{arcsec~pixel}^{-1}$ on both the red and blue chips, and a wavelength solution of $1.24 \AA \mathrm{pixel}^{-1}$ in the blue and $1.68 \AA \mathrm{pixel}^{-1}$ in the red. Measurement of arc lamp lines showed a FWHM resolution of $4.9 \AA$ on the blue chip and $7.1 \AA$ on the red chip. Worthey \& Ottaviani (1997) report that the resolution (FWHM) of the original Lick indices of interest as $8.4 \AA$. We therefore broaden our blue spectra with a Gaussian kernel with $\sigma=2.9 \AA$ to match the Lick system resolution.
Systematic and rotational velocities were removed by crosscorrelating a (logarithmically binned) stellar template plus Gaussian emission lines. The shifts were accurate to within 1 pixel $(\sim 1.2 \AA)$. The midplane rotation curve was used for both the midplane and off-plane spectra, as any difference between the two should be small at our resolution (Yoachim \& Dalcanton 2008).

After the two-dimensional spectra were broadened to match the Lick resolution, any foreground stars were masked and onedimensional spectra were extracted by summing in the spatial direction. For both the midplane and off-plane, we extracted spectra from the region of \pm 1 scale length $\left(h_{R}\right)$, as measured in Yoachim \& Dalcanton (2005).

We tested how the $\mathrm{S} / \mathrm{N}$ of our spectra affects the accuracy of the measured Lick indices. Adding artificial noise to template stellar spectra, we find the rms error in a measured Lick index scales with $\mathrm{S} / \mathrm{N}$ as $\sigma_{\text {index }} \sim 9(\mathrm{~S} / \mathrm{N})^{-1} \AA$. Therefore, to achieve an Lick

TABLE 1

Edge-On Galaxy Observations

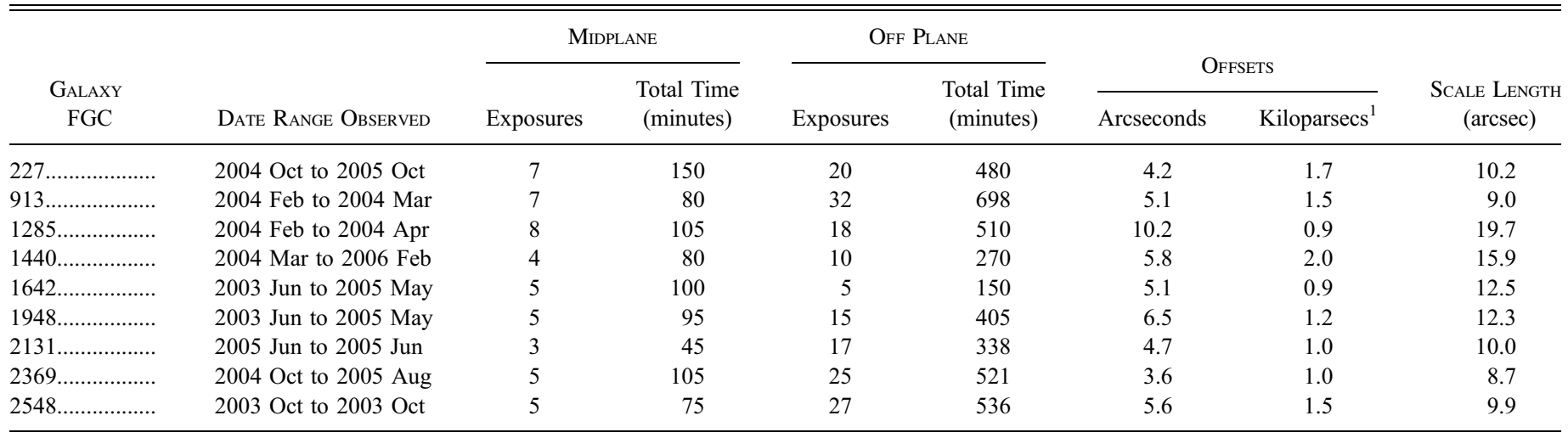

${ }^{1}$ Distances from Karachentsev et al. (2000). 


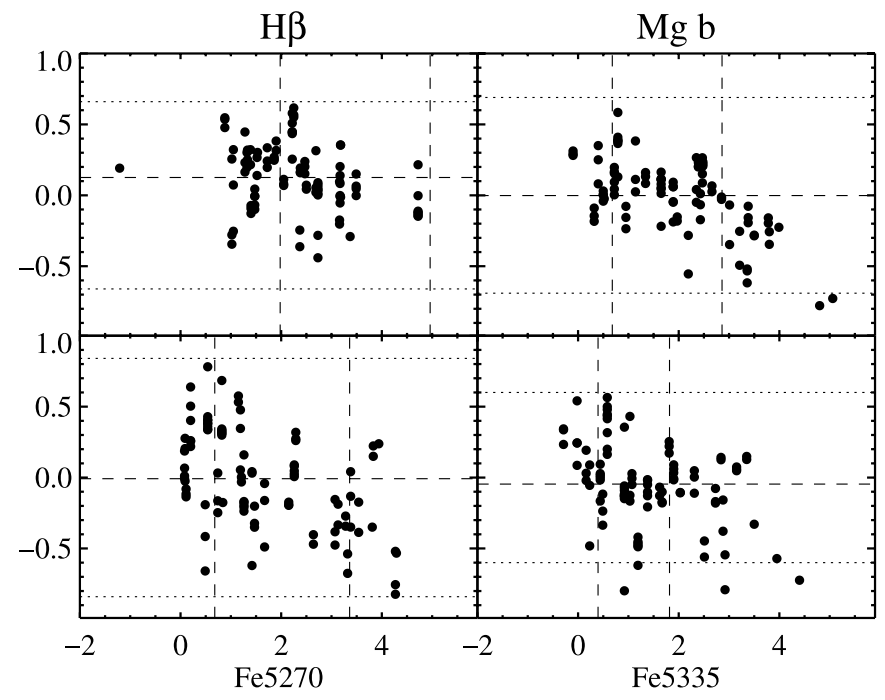

FIG. 2.-Comparison of Lick indices measured with DIS compared to the published equivalent widths in Worthey et al. (1994). Dashed horizontal lines show the mean offsets, while the dotted horizontal lines show the average Worthey et al. $\pm 3 \sigma$ uncertainties. Dashed vertical lines show the range of the line indices measured in our galaxy sample. Corresponding means and scatters are given in Table 3.

EW uncertainty of $\pm 0.2 \AA$ requires a S/N of $\sim 45$ per $\AA$, which agrees with previous determinations by Trager et al. (2000b).

Throughout our analysis, we calculate the uncertainties in our derived ages and metallicities using the $\mathrm{S} / \mathrm{N}$ of the extracted spectra. As an additional check, we have extracted one-dimensional spectra from $0<R / h_{R}<1$ and $-1<R / h-R<0$ to find the variance in the Lick indices from one side of the galaxy to the other and note cases where there are large discrepancies. This procedure allows us to flag systems where systematic errors are likely to dominate over random uncertainties.

\section{MOVING ONTO THE LICK SYSTEM}

While we have matched the Lick resolution reported in Worthey \& Ottaviani (1997) this is not enough to ensure that we are on the Lick system. Because the Lick indices were originally defined from spectra that had not been flux-calibrated, additional corrections are needed. To do so, we made 144 observations of 37 unique Lick standard stars. Stars for which we have repeat observations have a mean rms error of $0.09 \AA$ for each index. Our standard star EWs are compared to values listed in Worthey et al. (1994) and are shown in Figure 2. The derived zero-point corrections are listed in Table 2. In general, the agreement is quite good, with a typical Gaussian scatter of $0.26 \AA$ and little systematic offset. The notable exception is for $\mathrm{Mg} b$, for which the APO system measures low EW for metal rich systems with $\mathrm{Mg} b>3 \AA$. We do not explicitly correct for this offset as we are observing metal poor galaxies which have $\mathrm{Mg} b$ indices $<2 \AA$. We also compare our standard star EWs with stars in common with Schiavon (2007) and find a spread of $0.2-0.4 \AA$. Schiavon (2007) points out that measurements of EWs of bright stars are disturbingly inconsistent, and that there can be surprisingly large variations between observations. Schiavon (2007) argue that these offsets are caused by errors in the flat-fielding, which dominate the errors of the bright stars, unlike fainter galaxy spectra which are background limited.

We also observed a sample of elliptical galaxies which have reported Lick measurements in the literature. The ellipticals were observed when light cloud cover made observing faint regions of
TABLE 2

Zero-Point Conversions

\begin{tabular}{ccccr}
\hline \hline Index & $\mathrm{H} \beta$ & $\mathrm{Mg} b$ & $\mathrm{Fe} 5270$ & $\mathrm{Fe} 5335$ \\
\hline Zero point $^{\mathrm{a}}(\AA) \ldots \ldots \ldots \ldots . .$. & 0.13 & 0.00 & -0.01 & -0.05 \\
rms $(\AA) \ldots \ldots \ldots \ldots \ldots \ldots \ldots \ldots . .$. & 0.23 & 0.26 & 0.35 & 0.29 \\
\hline
\end{tabular}

${ }^{\text {a }}$ Value subtracted from our measured indices to move onto the Lick system.

disks impossible, or during brief periods when the primary targets were not visible. We observed nine galaxies in common with Trager et al. (2000b) listed in Table 3. Our indices are not directly comparable to the ones listed in Trager et al. (2000b) as we used our large $5^{\prime \prime}$ slit which samples more flux from the outer regions of the galaxies, and we have not replicated the corrections for velocity dispersion and emission-line fill-in as done in Trager et al. (2000b). Thus, some amount of scatter is expected. The residuals between our measurements and those in Trager et al. (2000b) are plotted in Figure 3. For the $\mathrm{H} \beta, \mathrm{Mg} b$, and Fe 5270 indices, we measure rms scatters of $0.16-0.31 \AA$ around the Trager et al. (2000b) values. There is a much larger scatter for Fe 5335 because the feature approaches the DIS dichroic cutoff for galaxies with a redshift greater than $4000 \mathrm{~km} \mathrm{~s}^{-1}$ and becomes very low $\mathrm{S} / \mathrm{N}$.

\subsection{Emission-Line Removal}

Unlike observations of gas-poor ellipticals and globular clusters, we must remove any emission lines that contaminate the index passbands before we can derive ages and metallicities from the absorption features. The removal of emission lines is not trivial. We would like to remove the $\mathrm{H} \beta$ emission, but to do so we would need to know the underlying shape of the stellar continuum. This shape in turn depends strongly on the stellar population's age and metallicity, which is what we set out to measure. We therefore must turn to other parts of the spectrum to estimate the amount of $\mathrm{H} \beta$ emission.

Lick indices, particularly $\mathrm{H} \beta$, are often contaminated with emission lines. González (1993) popularized using the correction $\mathrm{H} \beta_{\text {emission }}=0.7\left[\begin{array}{ll}\mathrm{O} & \mathrm{II}\end{array}\right] 25007$. This correction was derived by fitting a stellar template to the underlying spectrum of their elliptical galaxies to isolate the emission feature. Unfortunately, the shape of the template can determine the magnitude of the emission correction and the ratio of $F_{\mathrm{H} \beta} / F_{[\mathrm{O} \text { III }]}$ is highly metallicity sensitive (e.g., Kewley \& Dopita 2002), making this correction inappropriate for low-metallicity systems with varying stellar ages. Moorthy \& Holtzman (2006) make a correction by fitting a Gaussian to the emission peak inside the $\mathrm{H} \beta$ Lick index. This correction

TABLE 3

Elliptical Galaxies Observed

\begin{tabular}{ccc}
\hline Galaxy & Exposures & $\begin{array}{c}\text { Total Time } \\
\text { (minutes) }\end{array}$ \\
\hline NGC $1453 \ldots \ldots \ldots \ldots \ldots . \ldots \ldots$ & 2 & 25 \\
NGC $1600 \ldots \ldots \ldots \ldots \ldots$. & 2 & 20 \\
NGC $2778 \ldots \ldots \ldots \ldots \ldots$. & 3 & 9 \\
NGC $3379 \ldots \ldots \ldots \ldots \ldots$. & 1 & 5 \\
NGC $5638 \ldots \ldots \ldots \ldots \ldots$. & 2 & 10 \\
NGC $6127 \ldots \ldots \ldots \ldots \ldots$. & 2 & 20 \\
NGC $6702 \ldots \ldots \ldots \ldots \ldots$. & 2 & 10 \\
NGC $6703 \ldots \ldots \ldots \ldots \ldots$. & 2 & 20 \\
NGC $7052 \ldots \ldots \ldots \ldots \ldots$. & 1 & 5 \\
\hline
\end{tabular}



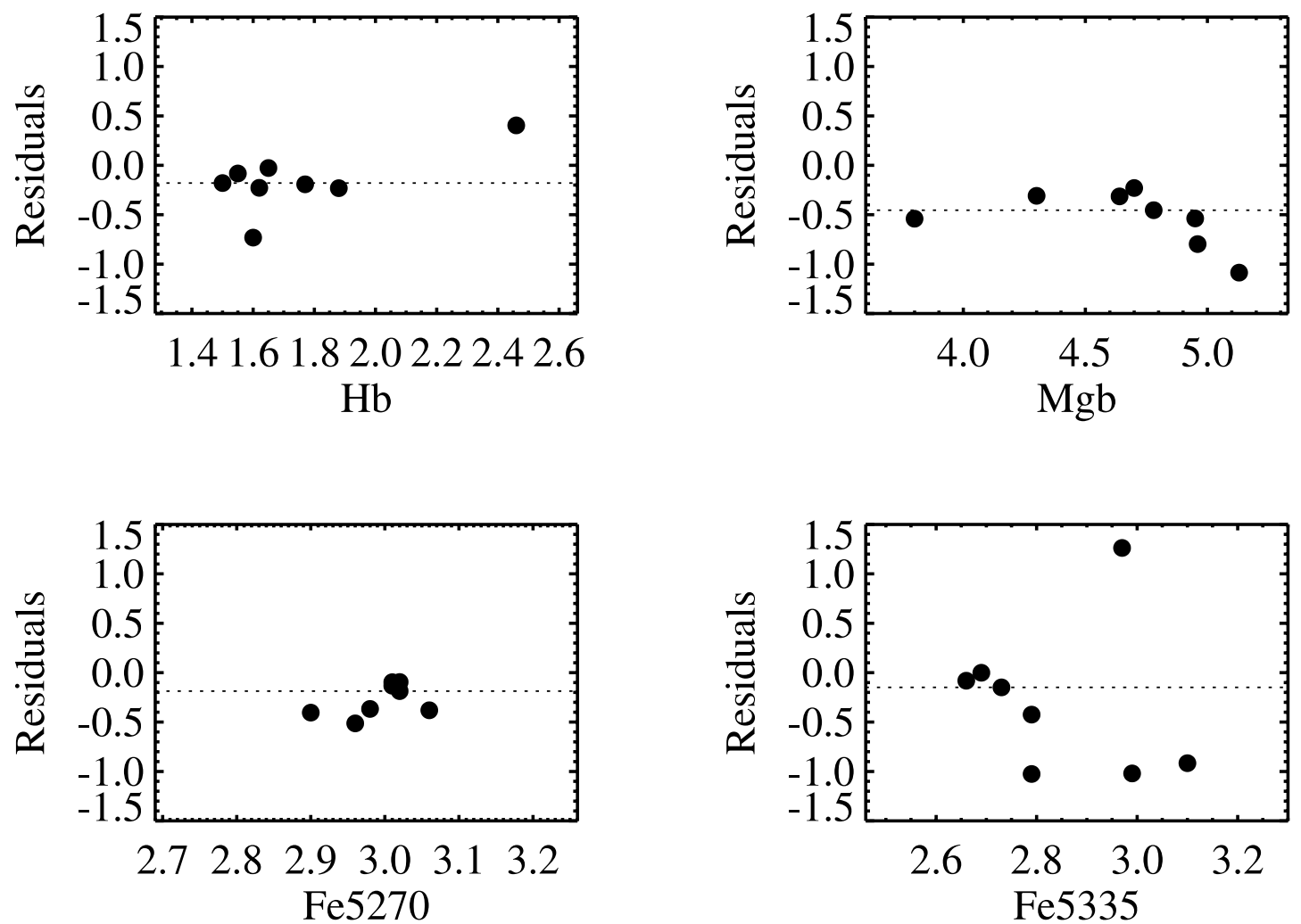

FIG. 3. - Lick indices measured with APO compared with values published in Trager et al. (2000b). Dashed lines show the median offset between our measurements and those in Trager et al. (2000b). The agreement is good, even with our larger slit and lack of velocity dispersion correction. The large errors for the Fe 5335 index values are caused by the feature approaching the DIS dichroic cutoff.

probably underestimates the $\mathrm{H} \beta$ correction since the Gaussian fit will not be sensitive to the fraction of $\mathrm{H} \beta$ emission that has filled the absorption feature. This correction is also very sensitive to the resolution of the measured spectrum. When we try to fit a Gaussian to the emission feature after broadening, we find that the correction is $0.4 \AA$ smaller than when we make the correction before broadening. MacArthur (2006) takes the most extreme measure of masking regions where the spectrum is contaminated by emission lines.

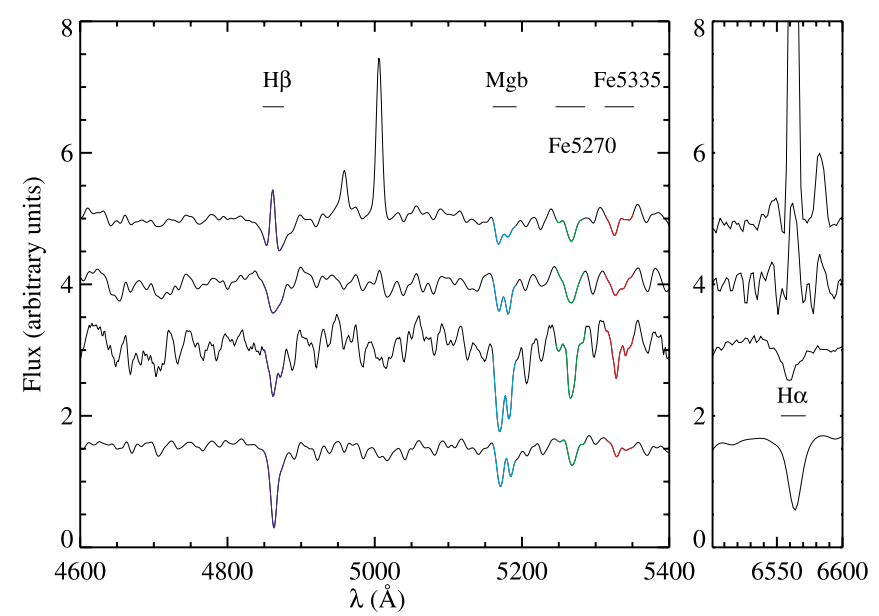

FIG. 4.-Examples of our blue and red spectra. From top to bottom: Midplane of FGC 1285, off-plane of FGC 1285, elliptical galaxy NGC 5638, and Lick standard star HD 114762. All the blue spectra have been smoothed to a resolution of $8.4 \AA$ and the Lick indices of interest have been labeled.
Using the [O III] emission line to estimate $\mathrm{H} \beta$ is a fine approximation when the $\mathrm{H} \beta$ emission correction is small, as in elliptical galaxy spectra. However, our midplane spectra are clearly dominated by the $\mathrm{H} \beta$ emission (Fig. 4), and we must use a more accurate technique to remove the emission. To do so, we measure the EW of the $\mathrm{H} \alpha$ emission line from the spectra taken simultaneously with the red DIS spectrograph and use this to estimate the $\mathrm{EW}$ of the $\mathrm{H} \beta$ emission line.

Our procedure for removing the $\mathrm{H} \beta$ emission is as follows. We first assume case B recombination with $F_{\mathrm{H} \alpha}=2.86 F_{\mathrm{H} \beta}$ (Osterbrock 1989). This correction is used in Rampazzo et al. (2005) and Denicoló et al. (2005) when measuring Lick indices in elliptical galaxies. However, because the $\mathrm{H} \alpha$ and $\mathrm{H} \beta$ emission lines were measured on different CCDs, we are hesitant to use the measured flux ratios. While both CCDs are calibrated using the same flux standard stars, the subsequent scaling and co-adding of frames was done independently and could skew the absolute flux calibration between the two. Instead, we make corrections based on equivalent widths as follows.

The definition of the EW is

$$
\mathrm{EW}=\int_{\lambda_{1}}^{\lambda_{2}}\left(1-\frac{F_{I \lambda}}{F_{C \lambda}}\right) d \lambda,
$$

where $F_{I \lambda}$ is the spectrum in the index passband and $F_{C \lambda}$ is the continuum spectrum calculated from the flanking pseudocontinuum regions. Our goal is to remove the contaminating emission feature to recover the equivalent width of the underlying Lick absorption feature:

$$
\mathrm{EW}_{\text {observed }}=\mathrm{EW}_{\text {Lick }}+\mathrm{EW}_{\text {emission }} .
$$


In the case where the continuum is constant, the EW from the emission alone is given by

$$
\mathrm{EW}_{\text {emission }}=\Delta \lambda-\frac{F_{\mathrm{em}}+F_{C \lambda} \Delta \lambda}{F_{C \lambda}}=-\frac{F_{\mathrm{em}}}{F_{C \lambda}},
$$

where $F_{\mathrm{em}}$ is the integrated flux in the emission line and $F_{C \lambda}$ is the continuum level. We can then write the expected EW ratio for the Balmer emission lines as

$$
\frac{\mathrm{EW}(\mathrm{H} \alpha)_{\mathrm{em}}}{\mathrm{EW}(\mathrm{H} \beta)_{\mathrm{em}}}=\frac{F_{\mathrm{H} \alpha}}{F_{\mathrm{H} \beta}} \frac{F_{C \lambda, \mathrm{H} \beta}}{F_{C \lambda, \mathrm{H} \alpha}} .
$$

This in turn can be modified to account for differential extinction between the stars and $\mathrm{H}$ in regions:

$$
\frac{\mathrm{EW}(\mathrm{H} \alpha)_{\mathrm{em}}}{\mathrm{EW}(\mathrm{H} \beta)_{\mathrm{em}}}=\frac{F_{\alpha}}{F_{\beta}} \frac{F_{C \lambda, \beta}}{F_{C \lambda, \alpha}} \frac{10^{0.4 E(B-V)_{\mathrm{gas}} k(\mathrm{H} \beta)-k(\mathrm{H} \alpha)}}{10^{0.4 E(B-V)_{\text {stars }} k(\mathrm{H} \beta)-k(\mathrm{H} \alpha)}} .
$$

Calzetti (2001) lists $k(\mathrm{H} \beta)-k(\mathrm{H} \alpha)=1.163$ and finds that $E(B-V)_{\text {stars }}=0.44 E(B-V)_{\text {gas }}$. The difference in reddening is due to the geometrically clumpy distribution of $\mathrm{H}$ II regions compared to the smoother distribution of stars. Finally, we get the relation

$$
\frac{\mathrm{EW}(\mathrm{H} \alpha)_{\mathrm{em}}}{\mathrm{EW}(\mathrm{H} \beta)_{\mathrm{em}}}=\frac{F_{\mathrm{H} \alpha}}{F_{\mathrm{H} \beta}} \frac{F_{C \lambda, \mathrm{H} \beta}}{F_{C \lambda, \mathrm{H} \alpha}} 10^{0.26 E(B-V)_{\mathrm{gas}}} .
$$

For the off-plane spectra, we assume there is negligible dust extinction, a fairly flat continuum level $\left(F_{C \lambda, \mathrm{H} \beta} \approx F_{C \lambda, \mathrm{H} \alpha}\right)$, and case $\mathrm{B}$ recombination, resulting in the standard correction of $\mathrm{EW}(\mathrm{H} \beta)_{\mathrm{em}}=y \mathrm{EW}(\mathrm{H} \alpha)_{\mathrm{em}}$ with $y=2.86$. In the case of the midplane spectra, there is a younger bluer stellar population and the possibility of dust extinction. We adopt case B recombination, a continuum ratio of 1.1 , and $E(B-V) \sim 0.1$ resulting in $y=3.3$. Our value of $E(B-V)$ is lower than typical values for the centers of edge-on disks (Matthews et al. (1999) find $E(B-$ $V) \sim 0.2$ in an edge-on disk similar to our sample galaxies), however, light off the midplane and at larger radii experience significantly less extinction. For elliptical galaxies identified as dusty, Denicoló et al. (2005) adopt a correction of $y=3.0$.

These adopted corrections give results consistent with what we expect from the broadband colors and SSP models. Most of the corrected $\mathrm{H} \beta$ Lick EWs fall within the range expected from the model grids, and we find younger ages where the integrated light is blue and older ages where it is red. In $\S 4.4$.1, we calculate how a $10 \%$ change in our adopted value of $y$ would propagate to the derived ages and metallicities. This uncertainty corresponds to extinction values $0.08<E(B-V)<0.42$ or continuum ratios $1.05<F_{C \lambda, \mathrm{H} \alpha} / F_{C \lambda, \mathrm{H} \beta}<1.3$.

While we consider how dust affects the emission-line EW ratios, the Lick absorption features themselves are fairly insensitive to dust (MacArthur 2005) and require no extra corrections even if the galaxies are dusty.

\subsection{Deriving Ages and Metallicities}

Ideally, we would fit full star formation and chemical evolution histories for our galaxies. However, we have chosen to focus on only four of the most prominent Lick indices, limiting the total number of parameters we can hope to fit. We therefore choose to interpret the spectra using SSP models. While our galaxies are clearly more complicated than single-burst stellar populations (given that we see old stellar populations along with signs of current star formation), the SSP models will still give a reasonable first-order approximation of the luminosity weighted mean ages, metallicities, and $\alpha$-element enhancements.

To convert our measured indices to ages we start with the model grids of Thomas et al. (2003) and interpolate them to a finer grid with age steps of $\delta t=0.1 \mathrm{Gyr}$ and metallicity steps of $\delta[Z / \mathrm{H}]=0.01$. We exclude the very young age grid points (age $<$ $0.1 \mathrm{Gyr})$ as the Lick indices expected for such young ages are degenerate with older populations. To calculate the uncertainties, we interpolate ages and metallicities for 100 Lick index pairs distributed according to the equivalent width uncertainties. We also test for possible systematic errors caused by incorrect emissionline corrections and calculate how a $10 \%$ error in the emissionline correction for $\mathrm{H} \beta$ propagates to errors in age and metallicity.

Figure 5 shows the Lick indices measured for each of our galaxies, placed on the model grids of Thomas et al. (2003) assuming no $\alpha$-enhancement. For each galaxy, we calculate the age and metallicity by interpolating onto grids of $\mathrm{H} \beta+\mathrm{Mg} b, \mathrm{H} \beta+$ Fe 5270, and $\mathrm{H} \beta+\mathrm{Fe} 5335$.

Three of the galaxies (FGC 1948, 2369, and 2548) have Lick indices that consistently fall far outside the model grids. This is not surprising, as the off-plane S/N for FGC 1948 and 2369 were so low we should not have expected to measure Lick indices (Table 4), and FGC 2548 is only on the cusp of having adequate $\mathrm{S} / \mathrm{N}$. For the remaining six galaxies, we plot the average interpolated ages and metallicities for the midplane and off-plane in Figure 6 and plot cumulative distributions of the age and metallicity differences between the thin- and thick-disk components in Figure 7. For consistency, we use only the metallicities measured from the $\mathrm{Mg} b$ and $\mathrm{Fe} 5270$ indices because several galaxies have very low $\mathrm{S} / \mathrm{N}$ at the Fe 5335 index.

For the six galaxies with high $\mathrm{S} / \mathrm{N}$, we find the thick disks have a median age of $7.2 \mathrm{Gyr}$ and metallicity of $[\mathrm{Z} / \mathrm{H}]=-0.6$. The thin disks have a similar median metallicity as expected for their low mass, but are uniformly much younger, with a median age of 4.6 Gyr. This age determination for the thin disk is derived after making very large emission-line corrections and represents a flux-weighted average of all the stars within a radius of 1 scale length. It is certainly possible that the oldest central regions of the thin disk contain stars whose ages are similar to those of the thick disks. We return to these results in $\S 4.3$ below.

\subsection{Radial Gradients}

The observations of the midplane of FGC 1440 were deep enough that we can extract radial gradients of the Lick indices. We extracted spectra by binning $6.3^{\prime \prime}$ spatially in a sliding region across the galaxy, and moving the observations onto the Lick system as before. This gives us a $\mathrm{S} / \mathrm{N}$ of 200 in the central regions of the galaxy and 50 at $1.3 h_{R}$. The radial variation of the Lick indices along with the interpolated ages and metallicities are plotted in Figures 8 and 9. The $\mathrm{H} \beta, \mathrm{Mg} b$, and $\mathrm{Fe} 5270$ indices have radial gradients of $2.8,-1.5$, and $-1.3 \AA / h_{R}$, respectively. These gradients are fairly large for pure disk systems. MacArthur (2006) finds similar gradients with similar magnitudes in four latetype face-on systems. Moorthy \& Holtzman (2006) also find strong radial gradients, but attribute them to the transition between bulge- and disk-dominated regions of their galaxies.

The gradients in the Lick indices are consistent with a large age gradient, with the central region of FGC 1440 having an SSP age of $\sim 12$ Gyr dropping to 2 Gyr after 1 scale length. The metallicity gradients, however, are slightly ambiguous. The metallicity measured from the $\mathrm{Mg} b$ index shows a mostly flat radial gradient, with a large drop on only one side of the galaxy. On the other hand the Fe 5270 index shows a decreasing metallicity on both sides of 
FGC1642 55km/s

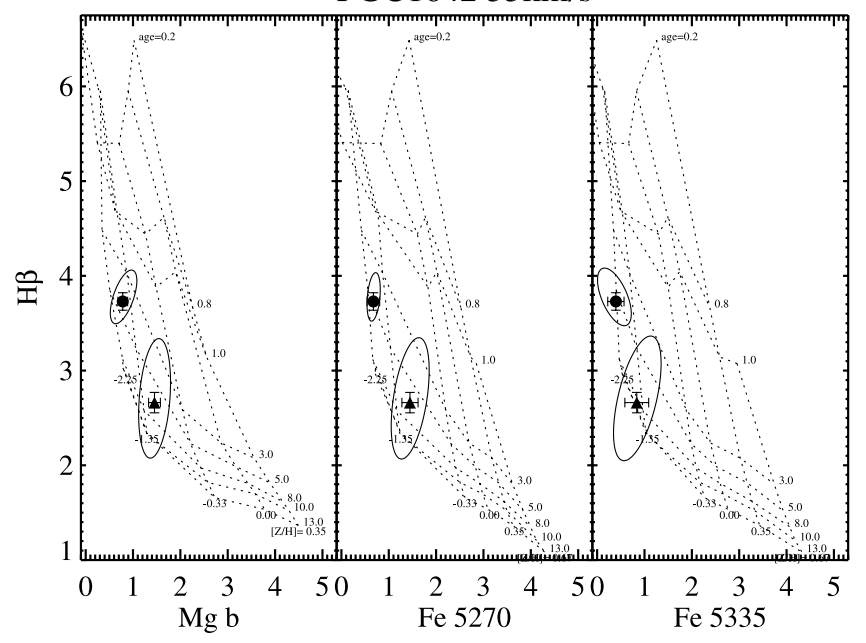

FGC1642

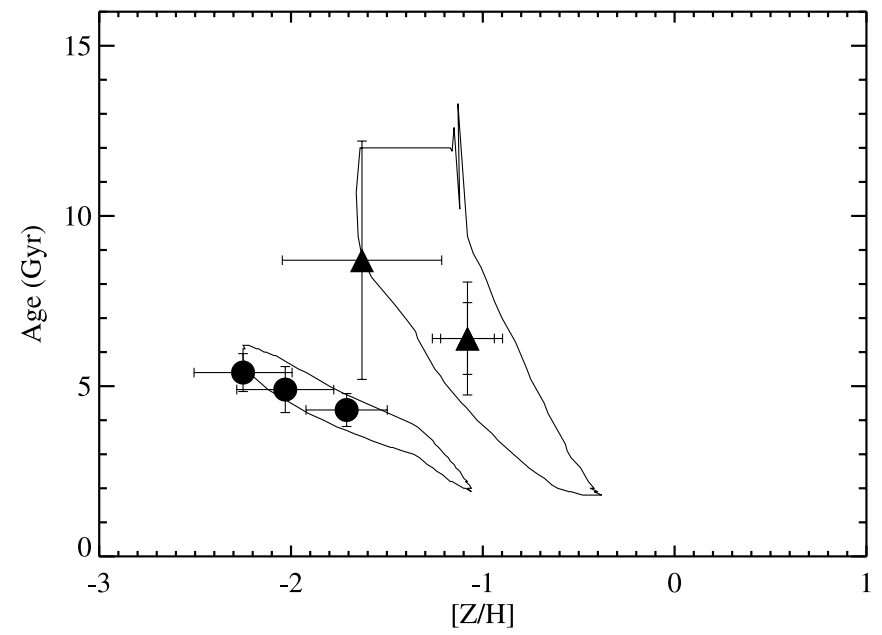

FIG. 5.-Lick index measurements for our observations. Top plots: Model grids from Thomas et al. (2003) along with our observed points. Blue points are from midplane observations and red points are for off-plane measurements measured from spectra extracted from $R<\left|h_{R}\right|$. Ellipses are drawn that encompass indices measured from the regions $0<R<h_{R}$ and $-h_{R}<R<0$. Small ellipses imply the measured indices are robust, while large ellipses show that the spectra on either side of the galaxy are not particularly consistent. Bottom plots: The interpolated ages and metallicities for all the above indices. Large filled points are used for spectra from the region $-h_{R}<R<h_{R}$ that fall inside the model grids. The ellipses from the $\mathrm{H} \beta-\mathrm{Mg} b$ plane are propagated to the bottom plots. Throughout, blue circles are used for midplane observations while red triangles are used for off-plane observations. Error bars on the points show the statistical uncertainties based on the $\mathrm{S} / \mathrm{N}$ of the spectra. [See the electronic edition of the Journal for a color version of this figure.]

the galaxy. Fitting a line to the radial data, we find that the $\mathrm{Mg} b$ index has a metallicity gradient of $-0.40 \pm 0.1 \mathrm{dex} / h_{R}$. If we restrict the fit to the region inside 1 scale length, the $\mathrm{Mg} b$ metallicity gradient is consistent with zero. The Fe 5270 index reveals a much steeper gradient of $-0.70 \pm 0.1 \mathrm{dex} / h_{R}$. This seems to imply that there is a radially changing level of $\alpha$-enhancement throughout the galaxy, with the central regions being close to solar composition and the outer regions becoming more $\alpha$-enhanced, thereby inflating the metallicity measured from $\mathrm{Mg} b$. In Figure 9 we plot the best fitting $\alpha$-element enhancement and it does appear that the outer regions are $\alpha$-enhanced compared to the central region which is best fit with a nearly solar chemical composition. These $\alpha$-element measurements should be regarded with caution,
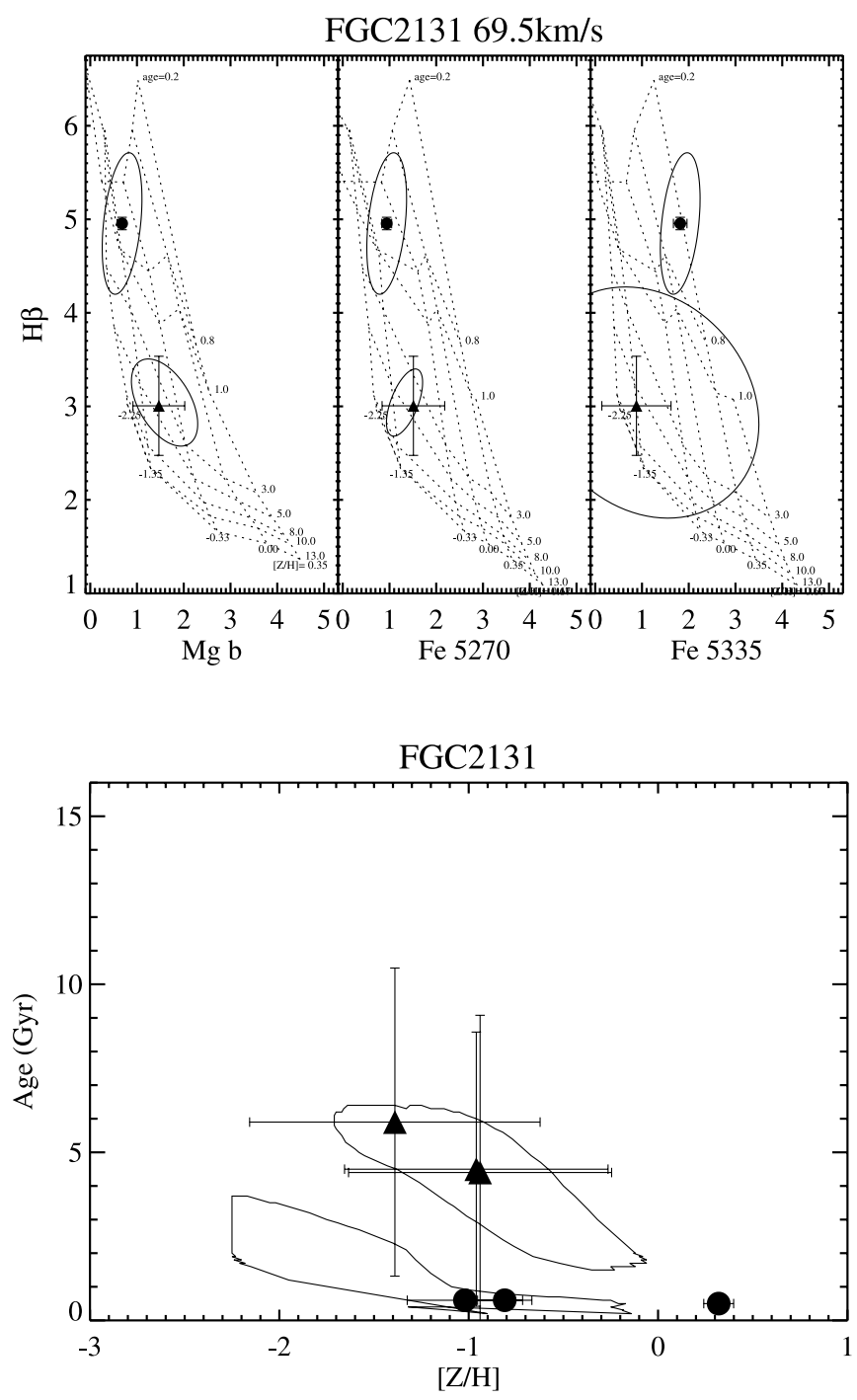

Fig. 5-Continued

as we have fit a model with three free parameters (age, metallicity, and $\alpha$-composition) using only three measurements $(\mathrm{H} \beta, \mathrm{Mg} b$, and Fe 5270). There is also the possibility that we should adopt a radially varying emission-line correction. This seems likely, as FGC 1440 hosts a dust lane which becomes less prominent with radius.

While we cannot draw broad conclusions based on a single galaxy, it is clear we detect stronger radial gradients than have been found in other disk systems. This is even more surprising given that edge-on projection effects should act to smooth any radial population gradients we observe. We discuss these results further in $\S 5.5$.

\subsection{Possible Uncertainties}

\subsubsection{Emission-Line Correction}

Both the midplane and off-plane have prominent emission lines. For the midplane spectra, we applied $\mathrm{H} \beta$ corrections that had an average of $4.9 \AA$, while the off-plane corrections had an average of $2.2 \AA$. If we had not take reddening or continuum shape into account and naively used just the case B recombination correction for both the midplane and off-plane, we would have derive slightly younger ages for the midplane. This simple correction would place many of the observations near the upper 
FGC1285 75km/s

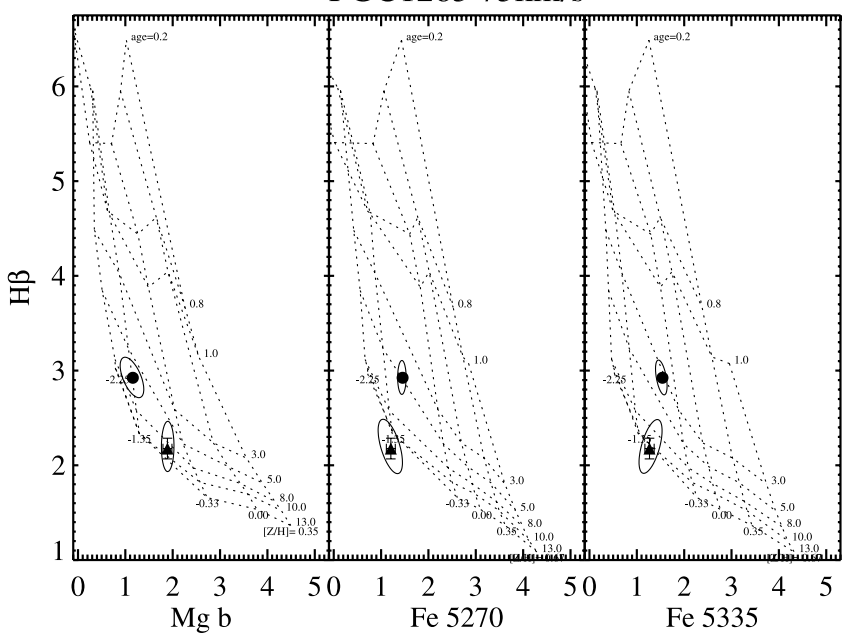

FGC1285

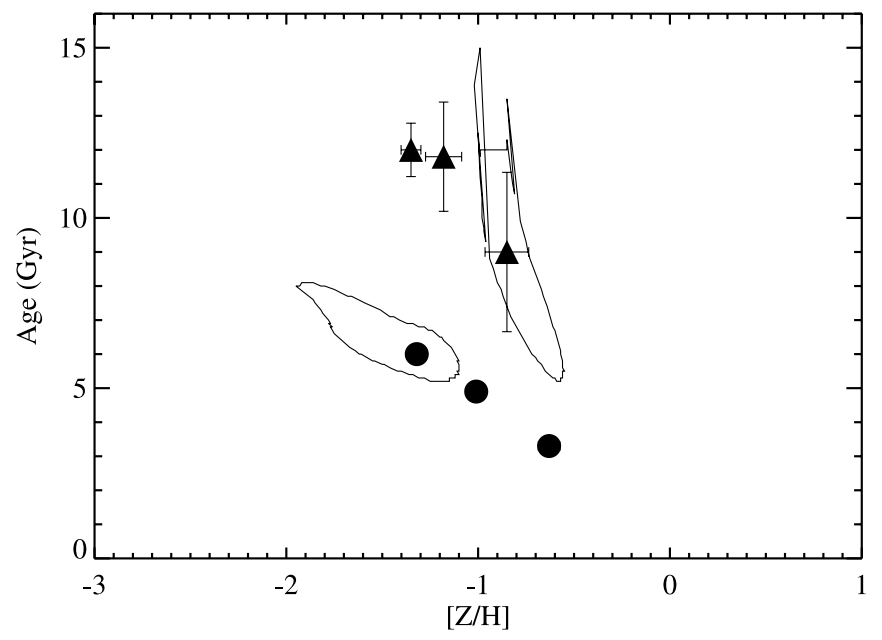

FGC913 77km/s

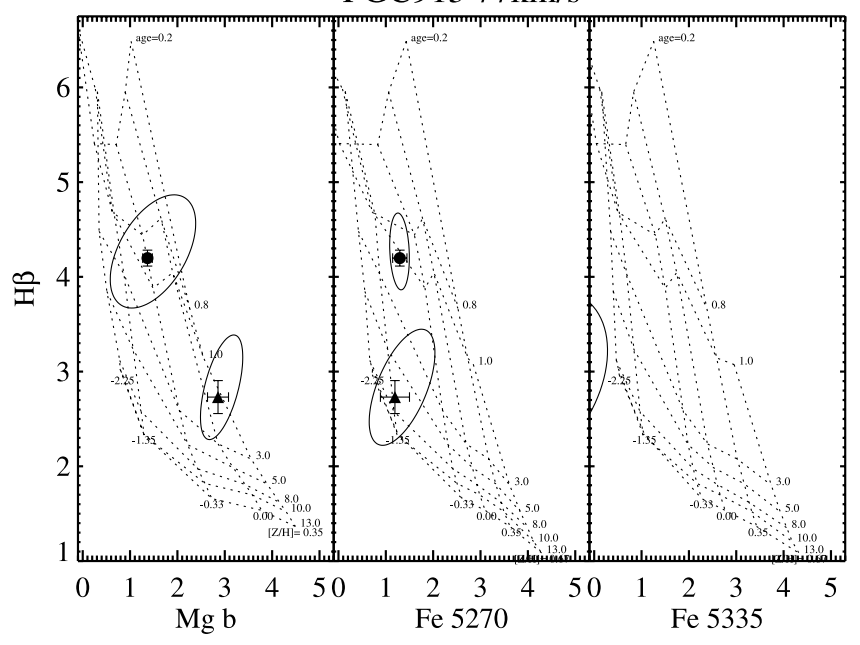

FGC913

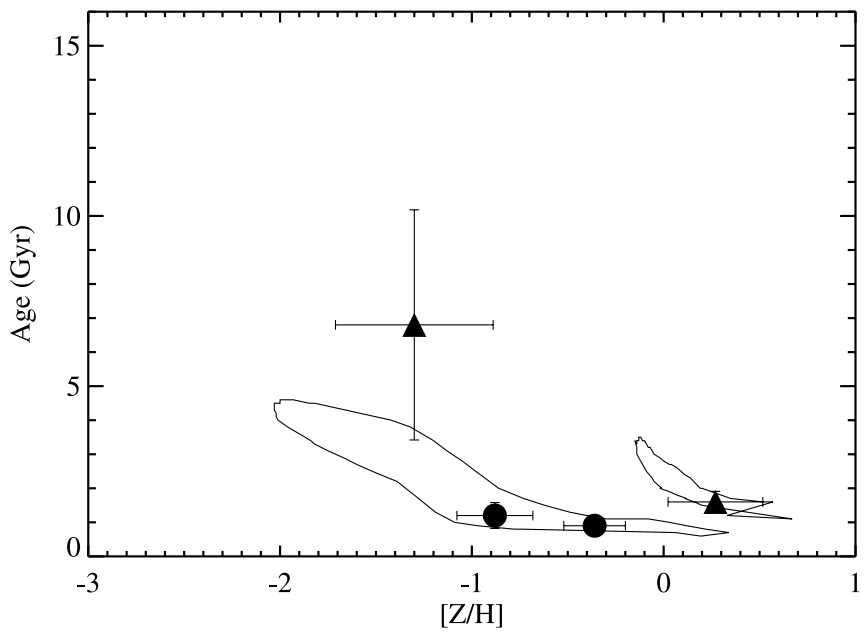

FIG. 5-Continued

edges of the model grids, suggesting we were under correcting. If instead we assume an even higher amount of dust extinction for the midplane, several of the thin and thick disks would then have similar SSP ages. However, it would take fairly extreme levels of dust extinction to drive all of the thin disks to have similar ages as the thick disks. The blue broadband colors for these galaxies are inconsistent with such high levels of dust.

Propagating an uncertainty of $\pm 10 \%$ in the $\mathrm{H} \beta$ emission correction results in a median $\mp 0.2$ dex shift in metallicity and \pm 1.9 Gyr in age for the midplane and $\mp 0.1$ dex and \pm 2.8 Gyr for the off-plane.

\subsubsection{Cross Contamination}

While we have placed our long slits at regions that should be dominated by the thin or thick components, we expect some thickdisk stars to be present in the midplane and vice-versa. Using the photometric fits in Yoachim \& Dalcanton (2006), we find that our midplane observations typically contain $\sim 20 \%$ thick-disk flux, while the off-plane observations have a flux contribution of $\sim 25 \%$ from the thin disk. As can be seen in Figure 1, we did not have large gaps between our slit positions. Observations made in poor seeing conditions might therefore experience extra cross-contamination as light from the midplane could be scattered into the off-plane slit position. This should not be a major problem as we avoided mak- ing off-plane observations during the worst seeing conditions, but could slightly increase the amount of expected cross-contamination.

Serra \& Trager (2007) study how Lick indices and their derived SSP ages and metallicities are affected when there are multiple stellar populations present. They find that the derived ages are very sensitive to the youngest stars present, while the metallicity measures are predominantly influenced by any older population. Contamination could therefore explain the similar metallicities we measure for the thin- and thick-disk stars. However, resolved stellar population studies have found small vertical metallicity gradients in low-mass galaxies as well (Seth et al. 2005). If enough thick-disk stars contaminate the midplane, our SSP derived metallicities will be slightly biased toward those of the older population, even in the young midplane. Of course, it is also possible that these low-mass galaxies have simply not undergone substantial star formation episodes and thus have not chemically enriched the thin disks above the level of the thick disk. Overall, the effects of cross-contamination would lead us to underestimate the true metallicity differences between the thin and thick disks, but to overestimate the flux-weighted age differences.

\subsubsection{Complex Stellar Populations}

While we are measuring SSP-equivalent ages and metallicities, it is fairly obvious that the midplanes of disk galaxies have 
FGC227 107km/s

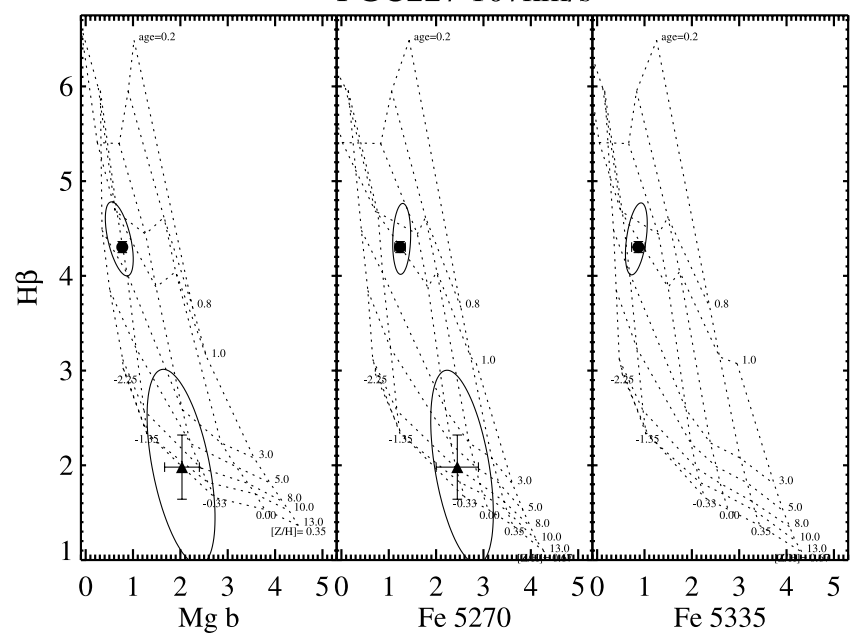

FGC227

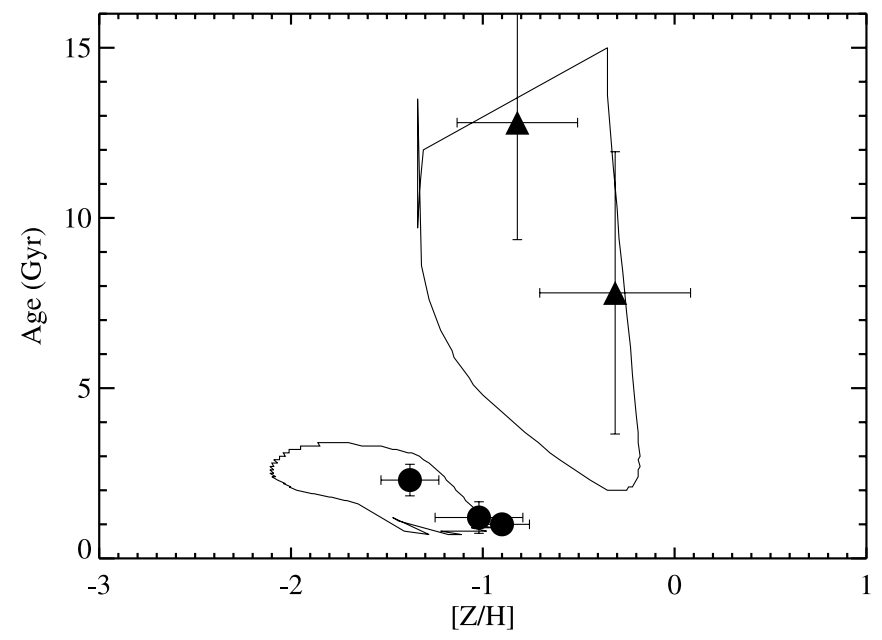

FGC1440 $150.5 \mathrm{~km} / \mathrm{s}$

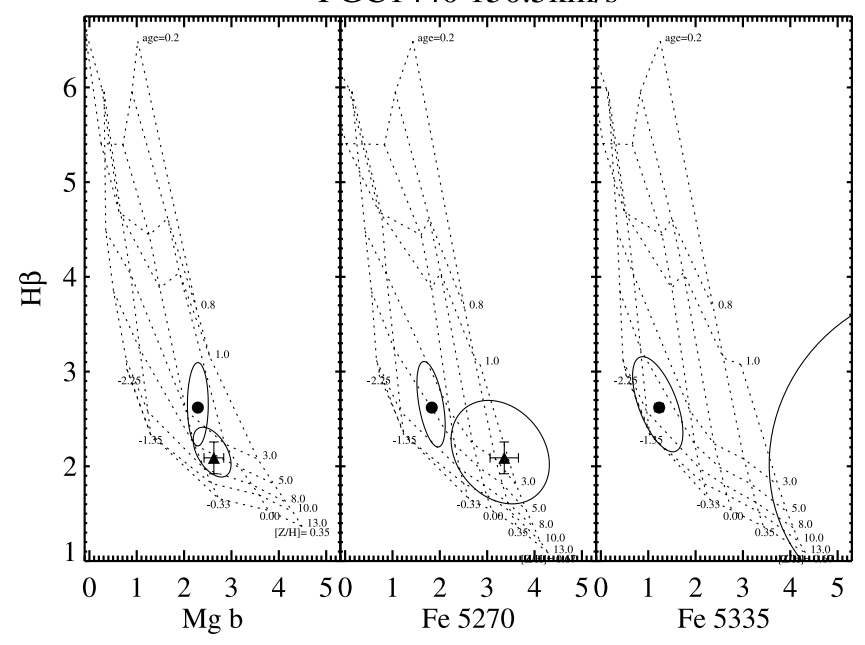

FGC1440

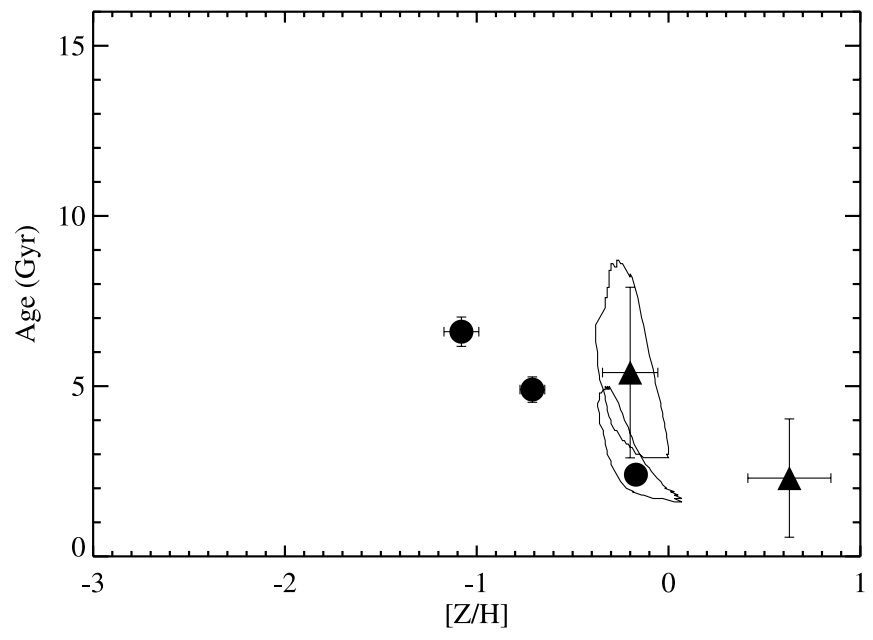

FIG. 5-Continued

undergone multiple epochs of star formation and are not well described by a single age and metallicity. Our ages and metallicities are thus best interpreted as flux-weighted averages across the extracted radial region, particularly for our midplane spectra where we have evidence for very steep radial age gradients in some systems. Because we are forced to bin our spectra over a large spatial region to reach adequate $\mathrm{S} / \mathrm{N}$, we include flux from the younger outer regions of the galaxy. Our midplane ages should probably be interpreted as minimums, as the central region is undoubtedly older than the flux-averaged measure we report.

\subsection{4. $\alpha$-Element Enhancement}

Many spectroscopic observations of elliptical galaxies and spiral bulges have found stellar populations that have systematic differences between the metallicity calculated from the $\operatorname{Mg} b$ index compared to the Fe indices. This systematic shift is usually interpreted as being caused by a stellar population that is significantly enhanced with $\alpha$-elements compared to the spectra that were used in building the Lick model grids. Such an enhancement is expected for stellar populations that form rapidly $(<1 \mathrm{Gyr})$ and that are primarily enriched by Type II supernovae (Matteucci 1994). Enhancements in $\alpha$-elements are often seen in elliptical galaxies (Worthey et al. 1992; Fisher et al. 1996; Thomas et al. 2003), as well as in local MW thick-disk stars (Bensby et al. 2005).
The galaxies in our sample are all fairly low mass and therefore also low metallicity. In the low-metallicity regime, the signature of $\alpha$-element enhancement becomes weaker in the Lick indices. Unlike massive elliptical galaxies where the metallicity indicators can show systematic offsets of $\sim 0.5$ dex for an $\alpha$-enhanced population, our galaxies are all subsolar metallicity and thus would show little bias even if they are $\alpha$-enhanced. If we used model SSP grids with $[\alpha / \mathrm{Fe}]=0.3$, our derived metallicities would change by only $\sim 0.1$ dex.

We are hesitant to use our data to fit the $\alpha$-element enhancement level. If we forge ahead and do so, we find considerable spread between the metallicities returned, but neither the thin or thick disk have systematically larger metallicities returned from the $\mathrm{Mg} b$ index, as we would expect if the stars were $\alpha$-enhanced. However, given the small expected offset and lower S/N than available for elliptical galaxy spectra, we do not consider this a significant result, and include it here only for completeness.

\section{DISCUSSION}

\subsection{Are Low-Mass Thick Disks Old?}

Measuring accurate ages for thick-disk stars has been done in relatively few systems. In the MW, stars that are kinematically identified as thick-disk stars typically have ages greater than $8 \mathrm{Gyr}$ 
TABLE 4

Measured Lick Indices in the Range $-1<h_{R}<1$

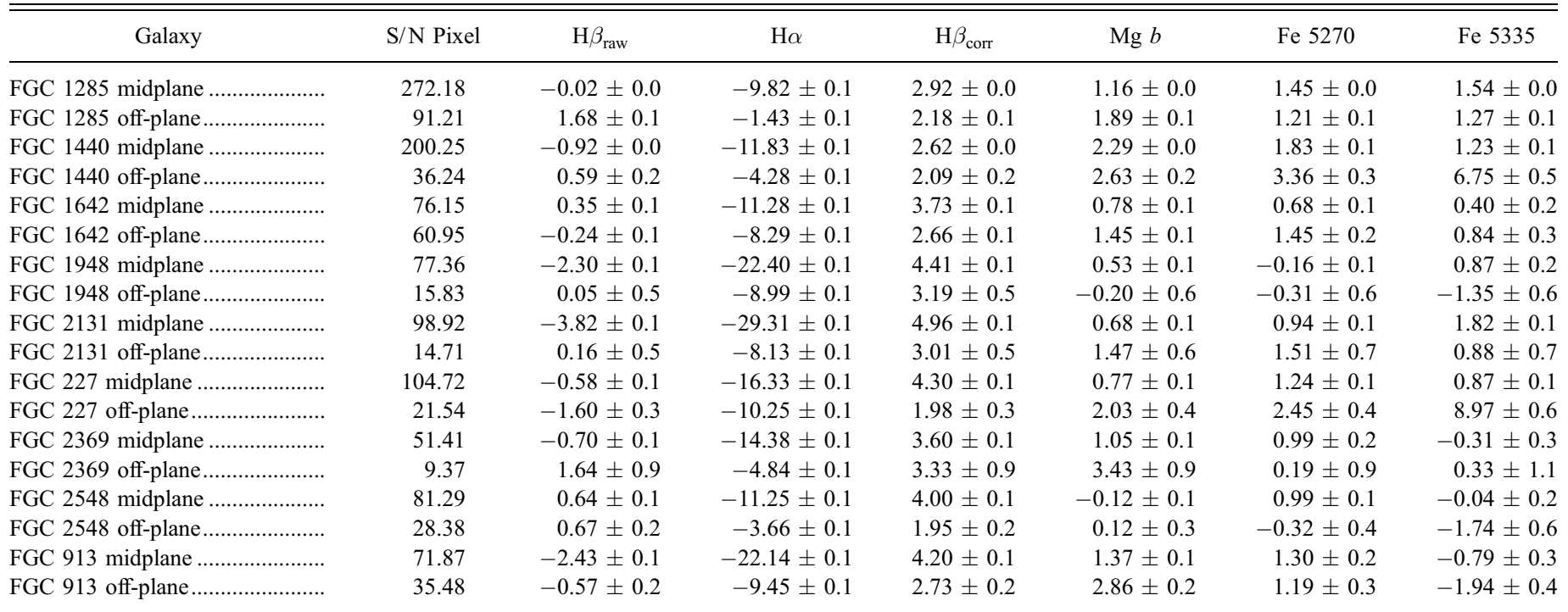

Notes.-All Lick index values are measured in angstroms. Negative values indicate emission.

(Fuhrmann 1998; Bensby et al. 2004b). HST studies of resolved stellar populations in nearby galaxies show the off-plane regions are dominated by old stars. Seth et al. (2005) find that in eight galaxies the off-plane RGB stars have ages in the range of 2-6 Gyr. Similarly, Mould (2005) uses the ratio of RGB and AGB stars to find ages of thick disks in a sample of four galaxies to be older than 3 Gyr. Our measured thick-disk SSP ages fall between 3.8 and 10.9 Gyr with a median age of 7.1 Gyr, consistent with these other studies that show thick disks to be dominated by ancient stars.

\subsection{Are Low-Mass Thick Disks Metal-Poor?}

Many of our thick disks appear to be more metal rich than the embedded thin disks. This counterintuitive result is probably due to the flux weighted nature of our measurement. The young, metal-poor outer regions dilute the true central ages and metallicities of the thin disks.

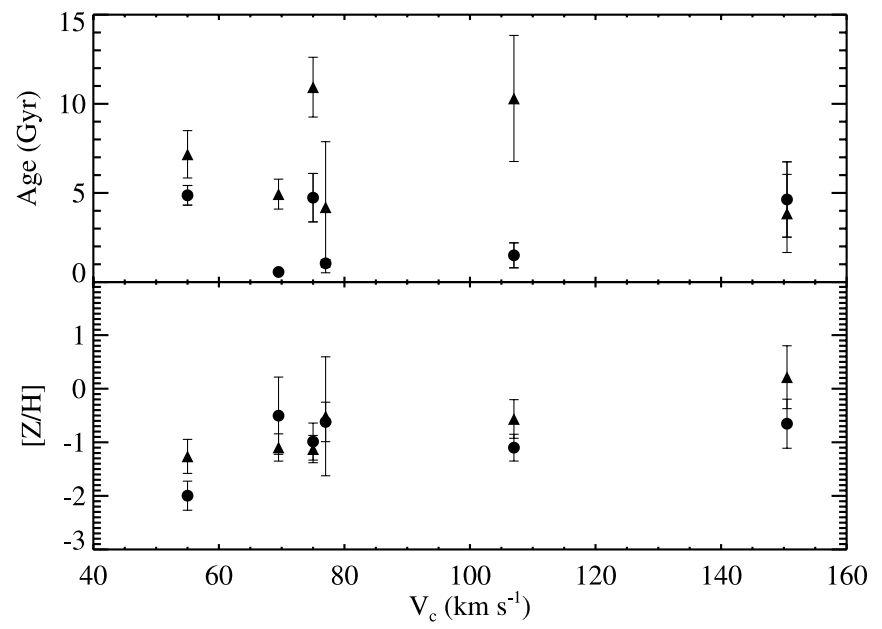

Fig. 6.-Average ages and metallicities as measured by Lick indices $\mathrm{H} \beta$, $\mathrm{Mg} b$, and Fe 5270. Blue circles show midplane (thin disk) observations while red triangles show off-plane (thick disk) observations. [See the electronic edition of the Journal for a color version of this figure.]
Thick-disk metallicities have only been measured for a handful of systems. MW thick-disk stars typically have metallicities in the range $[\mathrm{Fe} / \mathrm{H}] \sim-0.7$ to -0.2 , with the highest metallicity thick-disk stars possibly reaching solar values (Bensby et al. 2007). One difficulty with comparing to the MW thick disk is that the observed MW properties are of thick-disk stars near the solar radius, while we have only been able to measure thick-disk properties near the central regions of the galaxies. Fortunately there are signs that the MW thick disk has relatively small age and metallicity gradients (Bensby et al. 2005).

Constraints of thick-disk metallicities have also been derived from HST studies imaging resolved stars. Seth et al. (2005) used ACS images of six nearby edge-on disks to constrain vertical gradients in the stellar populations using the color and distribution of AGB and RGB stars. The older RGB stars have a systematically larger scale height compared to the younger AGB and mainsequence stars. They find little to no metallicity gradients in the thick-disk stars in their systems, with the metallicities of the thick

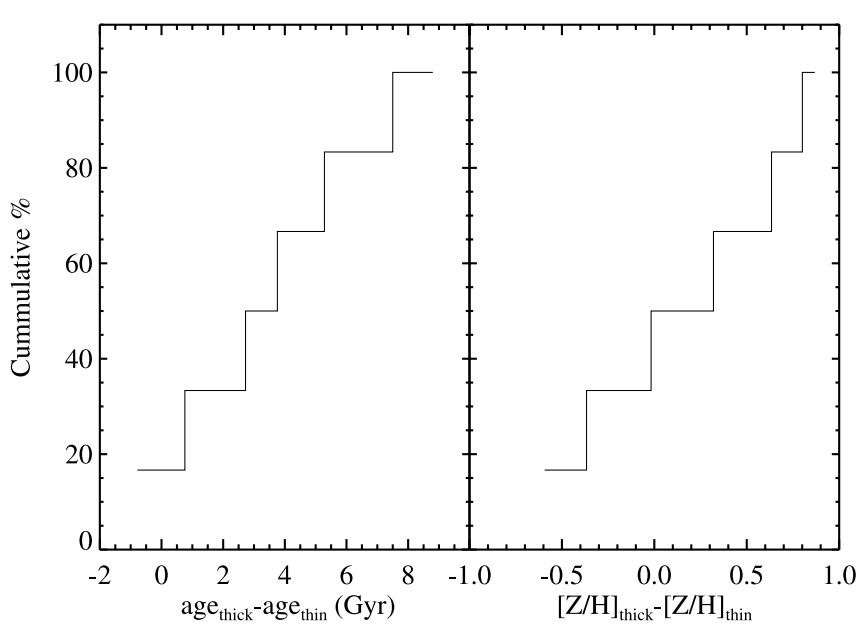

FIG. 7.-Cumulative distribution plots showing the differences between the thin-disk and thick-disk ages and metallicities. Positive ages mean the thick disk is older while negative metallicity differences mean the thick disk is metal poor in comparison to the thin disk. 

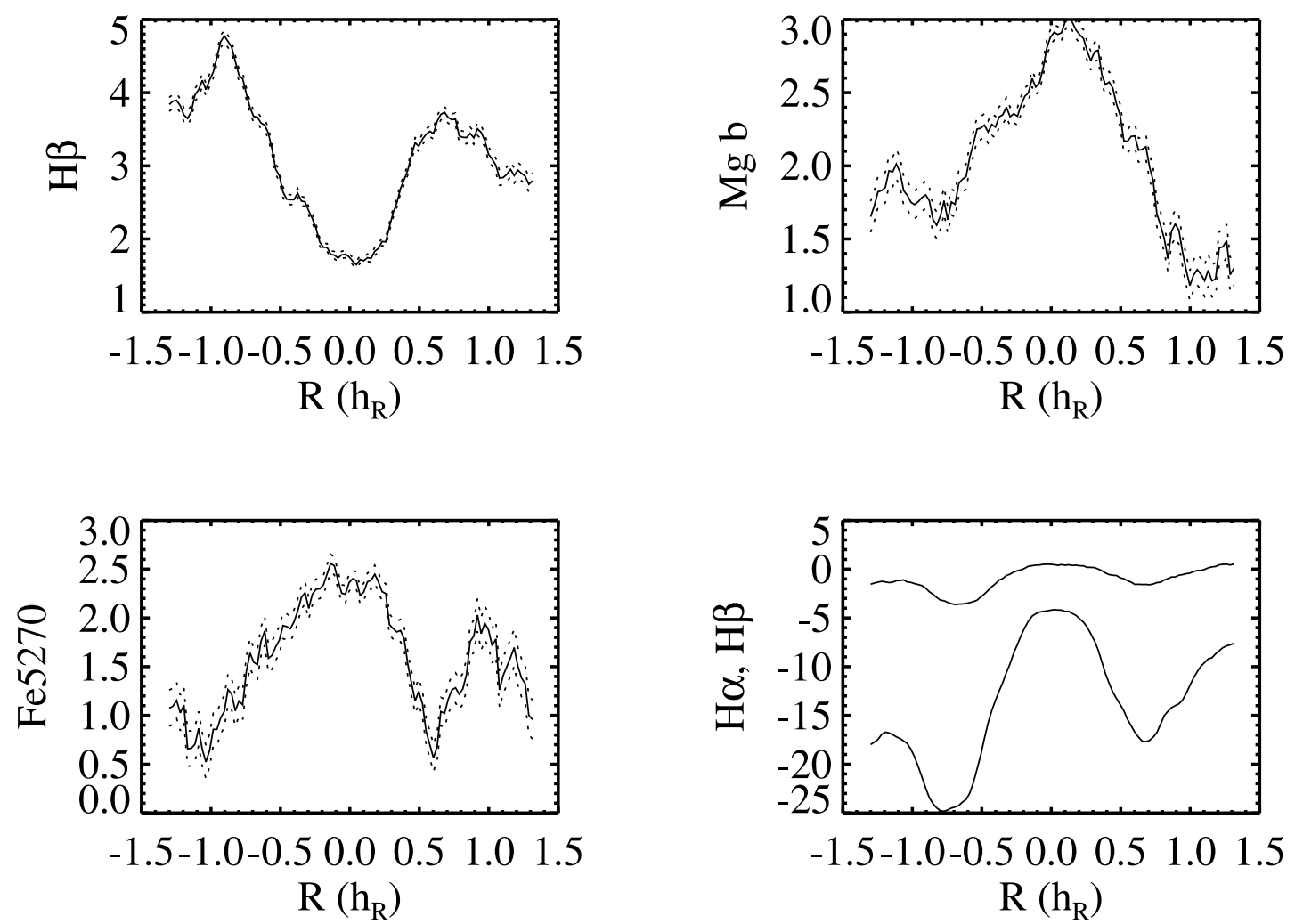

FIG. 8. - Radial gradients of the Lick equivalent widths measured in FGC 1440. The top left shows the $\mathrm{H} \beta$ index after correcting for emission-line fill-in, the top right shows $\mathrm{Mg} b$, the bottom left shows the Fe 5270 index, and the bottom right shows the uncorrected $\mathrm{H} \beta$ EW as well as the H $\alpha$ E. Dotted lines show the uncertainties calculated from the extracted spectra $\mathrm{S} / \mathrm{N}$.

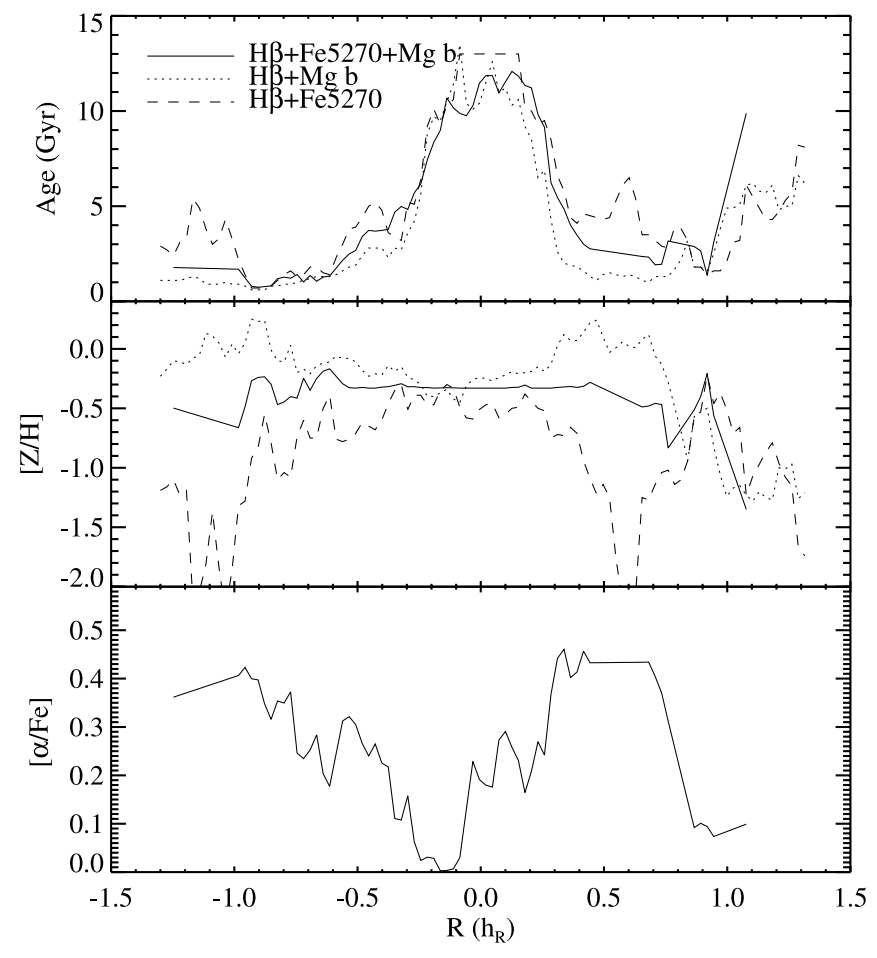

FIG. 9.- Interpolated age and metallicity measured along the midplane of FGC 1440. The solid line shows the best fit when the $\mathrm{H} \beta, \mathrm{Mg} b$, and $\mathrm{Fe} 5270$ indices are simultaneously fit to the best-matching Thomas et al. (2003) model. Dashed and dotted lines show the best fit if a solar composition is assumed. There is a very strong age gradient present. The $\mathrm{Mg} b$ index shows a fairly flat metallicity gradient, while the Fe 5270 index shows a radially decreasing metallicity. The third panel shows the best fitting $\alpha$-enhancement when we simultaneously fit all three indices. disks peaked around $[\mathrm{Fe} / \mathrm{H}] \sim-1$. This is slightly more metal poor than the measurements we have for our thick disks. However, the offset is unlikely to be significant, given that the Seth et al. (2005) study is able to study a cleaner sample of thick-disk stars by reaching higher vertical heights which reducing the contamination of thin-disk stars. Like the data presented here, Seth et al. (2005) only studies lower mass systems, limiting the amplitude of any possible metallicity gradient due to the low metallicity of the midplane. Using similar HST observations, Mould (2005) finds that thick-disk stars in four edge-on galaxies have $[\mathrm{Fe} / \mathrm{H}]$ between -1.0 and -0.78 , again very similar to the metallicities we find.

In Figure 10, we compare our thin-disk metallicities to the low-mass sample in Lee et al. (2006) and the large SDSS sample of Tremonti et al. (2004). We also compare our thick-disk values to thick-disk and halo samples presented in Seth et al. (2005); Mouhcine et al. (2005); Tikhonov et al. (2005) and Reddy et al. (2006). In cases where the authors presented values of $\log (\mathrm{O} / \mathrm{H})+$ 12 , we converted to $[\mathrm{Fe} / \mathrm{H}]$ assuming $\log (\mathrm{O} / \mathrm{H})_{\odot}+12=8.69$ and $[\mathrm{Fe} / \mathrm{O}]=0$. We also plot the midplane nebular abundances for our galaxies calculated from the S2N2 calibrator (Viironen et al. 2007). With the exception of a few outliers, our measured metallicities are consistent with metallicities measured in similar systems.

Recently, Ivezić et al. (2008) have questioned if the Milky Way thick disk is a unique component or simply an extension of the thin disk that has non-Gaussian metallicity and velocity distributions (Norris 1987). They cite a lack of correlation between velocity and metallicity in large SDSS samples as a major problem for traditional disk decompositions and rule out a "traditional" two-disk model at the $8 \sigma$ level. Unfortunately, the model Ivezić et al. (2008) rule out is not applicable to their observations. 


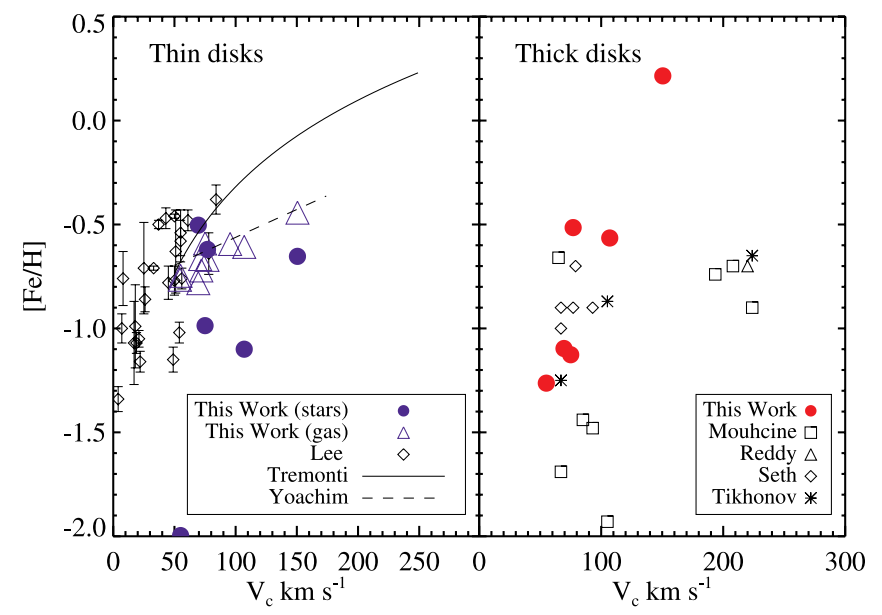

FIG. 10.-Comparison of our thin- and thick-disk metallicities to similar studies. The panel on the left shows our thin-disk metallicities along with the lowmass galaxies in Lee et al. (2006) and the fits to larger samples in Tremonti et al. (2004) and Yoachim \& Dalcanton (2007). On the right, we compare our thickdisk metallicities to the systems measured in Seth et al. (2005), Mouhcine et al. (2005), Reddy et al. (2006), and Tikhonov et al. (2005).

In particular, they model 1142 stars observed in the region $1.0<$ $z /$ kpc $<1.2$. Ivezić et al. (2008) correctly assume the observations will contain a similar number of thin- and thick-disk stars, however they do not use a realistic thin-disk component. The thindisk stars are modeled as having an asymmetric drift of $9 \mathrm{~km} \mathrm{~s}^{-1}$ and $[\mathrm{Fe} / \mathrm{H}]=-0.50$ with a spread of 0.04 dex (their Fig. 16). These parameters would be appropriate for modeling nearby thindisk stars, but they are observing stars 3-4 thin-disk scale heights above the Galactic plane. At this large height, only the kinematically hottest thin-disk stars will be present in the sample, and one should expect a much larger velocity lag than observed in local cooler thin-disk stars. Holmberg et al. (2007) show that the hottest local disk stars are the oldest and that the age-metallicity relation (AMR) for local stars has large intrinsic dispersion at large ages $(\sigma \sim 0.2 \mathrm{dex})$. This large metallicity dispersion is probably a result of radial migration of the older high velocity dispersion stars (Haywood 2008).

If the off-plane thin-disk stars are assumed to be similar to older local thin-disk stars (i.e., kinematically hot with a broad metallicity distribution and larger asymmetric drift), their properties should well match SDSS observations of regions at large scale height. Specifically, there should be little correlation between kinematics and metallicity despite the mixture of thin- and thick-disk stars. This revised model is consistent with the observations of thick disks presented here and elsewhere (Seth et al. 2005) that find stars at large scale heights are a significantly older population than those found near the midplane.

\subsection{Are Thick Disks $\alpha$-Enhanced?}

The level of $\alpha$-enhancement can be a major clue to the formation process of a stellar population. If $\alpha$-enhanced, it is a sign that a stellar population has been enriched mostly over a short time period by Type II SNe, whereas stars with solar composition formed over an extended period and have been enriched by both Type II and Type Ia SNe. Numerous papers have found that MW thick-disk stars are enhanced in $\alpha$-elements compared to thin-disk stars at similar total metallicities (e.g., Bensby et al. 2003, 2004a, 2005; Feltzing et al. 2003; Reddy et al. 2006; Tautvaišiené et al. 2001; Mashonkina et al. 2003; Prochaska et al. 2000; Fuhrmann $1998,2004)$. Unfortunately, we do not have the S/N to definitively say if our thick disks are $\alpha$-enhanced, due to the overall low metallicities of our target galaxies.

\subsection{Are Thick Disks "Normal” Stellar Populations?}

Using a sample of $>1000$ SDSS images of edge-on disk galaxies, Zibetti et al. (2004) examine the faint halo that appears when the images are stacked. This extended halo has anomalous colors, requiring stars that are either metal rich or have a bottom heavy IMF (Zibetti et al. 2004; Zackrisson et al. 2006). We find no such anomalies with the thick disks we observe spectroscopically, as most of them fall on stellar synthesis model grids using standard IMFs and metallicities. The few galaxies where we measure Lick indices that are inconsistent with the SSP models can easily be explained as spurious measurements caused by low $\mathrm{S} / \mathrm{N}$, and do not require exotic stellar populations.

\subsection{Radial Color Gradients in the Thin Disks}

Bell \& de Jong (2000) observed broadband colors for a large sample of galaxies and found that the radial gradients were predominantly caused by age gradients in the stellar populations. By averaging their sample together, They found a metallicity gradient, but broadband sensitivity to dust makes this a measurement in individual galaxies. Their work has been expanded on by MacArthur et al. (2004), who found both metallicity and age gradients are stronger in the inner regions of galaxies, and that galaxies with strong age gradients had smaller metallicity gradients. The Lick indices ability to lift the age-metallicity degeneracy, and relative insensitivity to dust makes it much easier to quantify how much of the radial color gradients in disk galaxies are due to age versus metallicity changes.

In one of the only study that has explicitly targeted Lick indices in the disk-dominated regions of galaxies, MacArthur (2006) detect age gradients in only two of their eight galaxies, and find strong negative metallicity gradients in four. The age gradients in MacArthur (2006) are also rather small $\left(-0.5\right.$ and $\left.-1.3 \mathrm{Gyr} / h_{R}\right)$. Our measurements of FGC 1440 show a much steeper age gradient, with the SSP age dropping by $\sim 9$ Gyr over 1 scale length.

Ganda et al. (2007) use the SAURON integral field unit spectrograph to measure Lick index strengths across the face of 18 late-type disk galaxies. They find in general $\mathrm{H} \beta$ increasing with radius and the metal-sensitive lines decreasing with radius. The SAURON observations show a very wide range of galaxy-togalaxy radial behavior, the most extreme are consistent with the strong gradients we find in FGC 1440.

Our finding that the thin disk of FGC 1440 might have stellar populations of near solar composition and be $\alpha$-enhanced at larger radii is puzzling. Looking at the transition between bulge- and disk-dominated regions, Moorthy \& Holtzman (2006) finds that the central bulges are either solar-composition or $\alpha$-enhanced, with little to no $\alpha$-enhancement in the disks. With the presence of old thick disks in all of the galaxies, we would expect the galaxies to have experienced plenty of chemical evolution and enrichment from SNe Ia. Instead, the outer regions of FGC 1440 are $\alpha$-enhanced, suggesting that the central region of the galaxy has undergone extended chemical enrichment, while the outer regions have not, despite being surrounded by old thick-disk stars.

This could be a sign that the thick-disk stars in FGC 1440 have been recently accreted, and thus have not contributed to the chemical enrichment the galaxy. Another possibility is that the central region of the galaxy is the only place where the gravitational potential is deep enough to retain SN ejecta, and the outer disk has historically suffered from SN blow-out and failed to retain metal enriched gas. Another possibility is that the luminosity-weighted 
metallicity in the outer disk is dominated by enrichment from the latest burst of star-formation which has $\alpha$-enhanced the region.

\section{CONCLUSIONS}

We have spectroscopically confirmed that the thick disks observed in edge-on late type galaxies are old, metal-poor stellar populations, analogous to the thick-disk stars seen in the MW and nearby edge-on systems. This is the first time ages and metallicities of thick disks have been measured in unresolved stellar populations. Because all of our targets are fairly low mass, we are unable to detect any significant differences between thin-disk and thick-disk metallicities. After correcting for emission-line contamination, the thin disks in our sample are found to be quite young, with strong radial age gradients.

We fail to detect any significant trend for thick-disk stars to be enhanced in $\alpha$-elements compared to their thin disks which is a defining characteristic of the MW thick disk. Our failure to observe $\alpha$-enhancements is most likely a result of our sample being dominated by low-mass and therefore low-metallicity galaxies, for which solar and $\alpha$-enhanced models are similar.

For one galaxy in our sample we have measured the radial gradients of the Lick indices in the thin disk and find the large gradients that are dominated by changes in the average stellar age with a small contribution from a changing average metallicity.

We thank Connie Rockosi and the UW machine shop for helping in the design and manufacture of our slit. We also thank the APO observing specialists for their help executing the observations. We thank Suzanne Hawley for reading an early version of this paper and making helpful comments. J. J. D. and P. Y. were partially supported through NSF grant CAREER AST 02-38683 and the Alfred P. Sloan Foundation. Based on observations obtained with the Apache Point Observatory $3.5 \mathrm{~m}$ telescope, which is owned and operated by the Astrophysical Research Consortium.
Abadi, M. G., Navarro, J. F., Steinmetz, M., \& Eke, V. R. 2003, ApJ, 597, 21 Abe, F., et al. 1999, AJ, 118, 261

Beauchamp, D., \& Hardy, E. 1997, AJ, 113, 1666

Bekki, K., \& Chiba, M. 2001, ApJ, 558, 666

Bell, E. F., \& de Jong, R. S. 2000, MNRAS, 312, 497

Bensby, T., Feltzing, S., \& Lundström, I. 2003, A\&A, 410, 527 2004a, A\&A, 415, 155 2004b, A\&A, 421, 969

Bensby, T., Feltzing, S., Lundström, I., \& Ilyin, I. 2005, A\&A, 433, 185

Bensby, T., Zenn, A. R., Oey, M. S., \& Feltzing, S. 2007, in ASP Conf. Ser. 374 , From Stars to Galaxies: Building the Pieces to Build Up the Universe, ed. A. Vallenari et al (San Francisco: ASP), 181

Benson, A. J., Lacey, C. G., Frenk, C. S., Baugh, C. M., \& Cole, S. 2004, MNRAS, 351, 1215

Brewer, M.-M., \& Carney, B. W. 2006, AJ, 131, 431

Brook, C. B., Kawata, D., Gibson, B. K., \& Freeman, K. C. 2004, ApJ, 612, 894

Bruzual, G., \& Charlot, S. 2003, MNRAS, 344, 1000

Burstein, D. 1979, ApJ, 234, 829

Burstein, D., Faber, S. M., Gaskell, C. M., \& Krumm, N. 1984, ApJ, 287, 586

Calzetti, D. 2001, PASP, 113, 1449

Chen, B., et al. 2001, ApJ, 553, 184

Chiba, M., \& Beers, T. C. 2000, AJ, 119, 2843

Dalcanton, J. J., \& Bernstein, R. A. 2000, AJ, 120, 203 2002, AJ, 124, 1328

de Grijs, R., \& Peletier, R. F. 1997, A\&A, 320, L21

de Grijs, R., \& van der Kruit, P. C. 1996, A\&AS, 117, 19

Denicoló, G., Terlevich, R., Terlevich, E., Forbes, D. A., Terlevich, A., \& Carrasco, L. 2005, MNRAS, 356, 1440

Faber, S. M., Friel, E. D., Burstein, D., \& Gaskell, C. M. 1985, ApJS, 57, 711

Feltzing, S., Bensby, T., \& Lundström, I. 2003, A\&A, 397, L1

Fisher, D., Franx, M., \& Illingworth, G. 1996, ApJ, 459, 110

Fuhrmann, K. 1998, A\&A, 338, 161 . 2004, Astron. Nachr., 325, 3

Ganda, K., et al. 2007, MNRAS, 380, 506

Gilmore, G., Wyse, R. F. G., \& Norris, J. E. 2002, ApJ, 574, L39

González, J. J. 1993, Ph.D. thesis, Univ. California (Santa Cruz)

Hänninen, J., \& Flynn, C. 2002, MNRAS, 337, 731

Hayashi, H., \& Chiba, M. 2006, PASJ, 58, 835

Haywood, M. 2008, MNRAS, in press (arXiv: 0805.1822)

Holmberg, J., Nordström, B., \& Andersen, J. 2007, A\&A, 475, 519

Ivezić, Z., et al. 2008, ApJ, in press (arXiv: 0804.3850)

Karachentsev, I. D., Karachentseva, V. E., Kudrya, Y. N., Makarov, D. I., \& Parnovsky, S. L. 2000, Bull. Spec. Astrophys. Obs., 50, 5

Karachentsev, I. D., Karachentseva, V. E., \& Parnovskij, S. L. 1993, Astron. Nachr., 314, 97

Kazantzidis, S., Bullock, J. S., Zentner, A. R., Kravtsov, A. V., \& Moustakas, L. A. 2007, preprint (arXiv: 0708.1949)

Kewley, L. J., \& Dopita, M. A. 2002, ApJS, 142, 35

Kroupa, P. 2002, MNRAS, 330, 707

Lee, H., Skillman, E. D., Cannon, J. M., Jackson, D. C., Gehrz, R. D., Polomski, E. F., \& Woodward, C. E. 2006, ApJ, 647, 970

MacArthur, L. A. 2005, ApJ, 623, 795

2006, Ph.D. thesis, Univ. British Columbia

\section{EFERENCES}

MacArthur, L. A., Courteau, S., Bell, E., \& Holtzman, J. A. 2004, ApJS, 152, 175 Martin, N. F., Ibata, R. A., Bellazzini, M., Irwin, M. J., Lewis, G. F., \& Dehnen, W. 2004, MNRAS, 348, 12

Mashonkina, L., Gehren, T., Travaglio, C., \& Borkova, T. 2003, A\&A, 397, 275

Matteucci, F. 1994, A\&A, 288, 57

Matthews, L. D., Gallagher, J. S., \& van Driel, W. 1999, AJ, 118, 2751

Moorthy, B. K., \& Holtzman, J. A. 2006, MNRAS, 371, 583

Morrison, H. L., Miller, E. D., Harding, P., Stinebring, D. R., \& Boroson, T. A. 1997, AJ, 113, 2061

Mouhcine, M., Rich, R. M., Ferguson, H. C., Brown, T. M., \& Smith, T. E. 2005, ApJ, 633, 828

Mould, J. 2005, AJ, 129, 698

Navarro, J. F., Helmi, A., \& Freeman, K. C. 2004, ApJ, 601, L43

Neeser, M. J., Sackett, P. D., De Marchi, G., \& Paresce, F. 2002, A\&A, 383, 472

Norris, J. 1987, ApJ, 314, L39

Osterbrock, D. E. 1989, Astrophysics of Gaseous Nebulae and Active Galactic Nuclei (Mill Valley: University Science Books)

Osterbrock, D. E., Fulbright, J. P., Martel, A. R., Keane, M. J., Trager, S. C., \& Basri, G. 1996, PASP, 108, 277

Peletier, R. F., et al. 2007, MNRAS, 379, 445

Pohlen, M., Balcells, M., Lütticke, R., \& Dettmar, R.-J. 2004, A\&A, 422, 465 Prochaska, J. X., Naumov, S. O., Carney, B. W., McWilliam, A., \& Wolfe, A. M. 2000, AJ, 120, 2513

Proctor, R. N., Sansom, A. E., \& Reid, I. N. 2000, MNRAS, 311, 37

Prugniel, P., Maubon, G., \& Simien, F. 2001, A\&A, 366, 68

Quinn, P. J., Hernquist, L., \& Fullagar, D. P. 1993, ApJ, 403, 74

Rampazzo, R., Annibali, F., Bressan, A., Longhetti, M., Padoan, F., \& Zeilinger, W. W. 2005, A\&A, 433, 497

Read, J. I., Lake, G., Agertz, O., \& Debattista, V. P. 2008, MNRAS, in press (arXiv:0803.2714)

Reddy, B. E., Lambert, D. L., \& Allende Prieto, C. 2006, MNRAS, 367, 1329 Reid, N., \& Majewski, S. R. 1993, ApJ, 409, 635

Robin, A. C., Haywood, M., Creze, M., Ojha, D. K., \& Bienayme, O. 1996, A\&A, 305, 125

Ryder, S. D., Fenner, Y., \& Gibson, B. K. 2005, MNRAS, 358, 1337

Sánchez-Blázquez, P., Forbes, D. A., Strader, J., Brodie, J., \& Proctor, R. 2007, MNRAS, 377, 759

Schiavon, R. P. 2007, ApJS, 171, 146

Serra, P., \& Trager, S. C. 2007, MNRAS, 374, 769

Seth, A. C., Dalcanton, J. J., \& de Jong, R. S. 2005, AJ, 130, 1574

Shaw, M. A., \& Gilmore, G. 1989, MNRAS, 237, 903

Statler, T. S. 1988, ApJ, 331, 71

Tautvaišienè, G., Edvardsson, B., Tuominen, I., \& Ilyin, I. 2001, A\&A, 380, 578

Thomas, D., Maraston, C., \& Bender, R. 2003, MNRAS, 339, 897

Tikhonov, N. A., \& Galazutdinova, O. A. 2005, Astrophys., 48, 221

. 2008, Astron. Rep., 52, 19

Tikhonov, N. A., Galazutdinova, O. A., \& Drozdovsky, I. O. 2005, A\&A, 431, 127

Trager, S. C., Faber, S. M., Worthey, G., \& González, J. J. 2000a, AJ, 120, 165 2000b, AJ, 119, 1645 
Trager, S. C., Worthey, G., Faber, S. M., Burstein, D., \& Gonzalez, J. J. 1998, ApJS, 116, 1

Tremonti, C. A., et al. 2004, ApJ, 613, 898

Tsikoudi, V. 1979, ApJ, 234, 842

van der Kruit, P. C. 1984, A\&A, 140, 470

van Dokkum, P. G., Peletier, R. F., de Grijs, R., \& Balcells, M. 1994, A\&A, 286,415

van Zee, L., Salzer, J. J., Haynes, M. P., O’Donoghue, A. A., \& Balonek, T. J. 1998, AJ, 116, 2805

Vazdekis, A., \& Arimoto, N. 1999, ApJ, 525, 144

Velazquez, H., \& White, S. D. M. 1999, MNRAS, 304, 254

Viironen, K., Delgado-Inglada, G., Mampaso, A., Magrini, L., \& Corradi, R. L. M. 2007, MNRAS, 381, 1719

Villalobos, Á., \& Helmi, A. 2008, preprint (arXiv: 0803.2323)

Villumsen, J. V. 1985, ApJ, 290, 75
Walker, I. R., Mihos, J. C., \& Hernquist, L. 1996, ApJ, 460, 121 Worthey, G. 1994, ApJS, 95, 107

Worthey, G., Faber, S. M., \& Gonzalez, J. J. 1992, ApJ, 398, 69

Worthey, G., Faber, S. M., Gonzalez, J. J., \& Burstein, D. 1994, ApJS, 94, 687

Worthey, G., \& Ottaviani, D. L. 1997, ApJS, 111, 377

Wu, H., et al. 2002, AJ, 123, 1364

Yoachim, P., \& Dalcanton, J. J. 2005, ApJ, 624, 701 2006, AJ, 131, 226

2007, BAAS Meeting Abstracts, 211, 95.18 2008, ApJ, 682, 1004

Zackrisson, E., Bergvall, N., Östlin, G., Micheva, G., \& Leksell, M. 2006, ApJ, 650,812

Zaritsky, D., Kennicutt, Jr., R. C., \& Huchra, J. P. 1994, ApJ, 420, 87 Zibetti, S., White, S. D. M., \& Brinkmann, J. 2004, MNRAS, 347, 556 